\title{
INTERPRETATION OF THE X-RAY SPECTRA OF SOLAR ACTIVE REGIONS*
}

\author{
ARTHUR B. C. WALKER, JR. \\ Institute for Plasma Research and Department of Applied Physics, \\ Stanford University, Stanford, Calif., U.S.A.
}

\begin{abstract}
This paper presents a review of recent analytical studies of the coronal X-ray spectrum below $25 \AA$. The techniques used to compute the theoretical coronal spectrum, and the currently available atomic rate constant data are reviewed first. Spectroscopic techniques which have been proposed for the determination of coronal temperature and density structure, and the results derived from their application to coronal spectra are also reviewed.

A number of coronal models based on X-ray observations have been developed recently, and the coronal temperature structure and composition predicted by these models is discussed, and compared with models' of the corona and transition region derived from studies of the solar EUV spectrum.
\end{abstract}

\section{Introduction}

In this review the interpretation of the soft X-ray spectrum of the solar corona, and the development of models of coronal composition and density and temperature structure will be discussed.

Comprehensive reviews of spectroscopic observations of the corona in the grazing incidence region of the spectrum $(\lambda \lesssim 300 \AA)$, and of the techniques used to interpret these observations, were presented at the Third Symposium on Ultraviolet and X-ray Spectroscopy of Astrophysical and Laboratory Plasmas by Doschek (1972) for flare observations, and by Walker (1972) for the non-flaring Sun. Culhane and Acton (1974) have reviewed more recent work.

In the preceding paper Dr Parkinson (1975) has reviewed the observational techniques used, and the results of recent spectroscopic studies of the coronal spectrum below $25 \AA$. In the present paper, the analytical techniques used to interpret the coronal X-ray spectrum, and the coronal models derived with these techniques will be emphasized.

\section{Techniques for the Construction of Coronal Models from Spectroscopic Observations}

Walker (1972) and Gabriel and Jordan (1972) have reviewed the calculation of the coronal spectrum, and the spectroscopic techniques which have been used to construct models of the corona. Consequently, I shall only briefly summarize the status of calculations of the coronal spectrum, with emphasis on the most recent results. The spectroscopic techniques used for the development of coronal models have also been adequately treated in the above references, and a brief summary will suffice here.

The development of coronal models from coronal permitted line observations was first discussed in a general way by Pottasch (1964, 1966, 1967). Pottasch's analysis

* This work was supported by NASA Grant, NGR 05-020-695. 
was confined to transition region lines whose excitation functions have a sharply peaked dependence on temperature, and he was able to replace the explicit temperature dependence of the emission function of each line by an average emission function evaluated at the temperature of most efficient emission for that line. A number of authors (Batstone et al. (1970), Chambe (1971) and Walker (1972)) have revised Pottasch's analysis for coronal lines, by including the temperature variation of each line explicitly. Walker et al. (1974a) have presented a systematic formulation of this approach. Since the spectroscopically resolved observations of the corona which have been available prior to the launch of the Apollo Telescope Mount (ATM) solar observatory on Skylab have not had sufficient spatial resolution to allow the physical structure of coronal X-ray emitting regions to be studied, analyses of these observations have ignored the geometry of the emitting region. The observation of X-ray emitting arch filament systems by Skylab (Vaiana et al., 1973; Vaiana, 1974; Underwood, 1974) has emphasized the importance of the magnetic structure of coronal active regions. Accordingly, I will conclude this section with a discussion of the modification in the analytical approach developed by Pottasch required to take account of the geometrical constraints imposed by the coronal magnetic structure.

\section{(a) CALCULATION OF THE CORONAL LINE SPECTRUM}

The intensity observed in an emission line (with upper level $i$ and lower level $j$ ) in ionization stage $z$ of element $Z$ is given by

$$
I_{Z z i j}=\iiint E_{Z z i j}\left[T_{e}\left(x_{v}\right), n_{e}\left(x_{v}\right)\right] \mathrm{d} x_{1} \mathrm{~d} x_{2} \mathrm{~d} x_{3}, \quad v=1,2,3 .
$$

The integral is over the coronal volume unresolved by the spectroscopic observation.

The emission function for a particular line may be written

$$
E_{Z z i j}=\frac{h c}{\lambda_{i j}} A_{i j}^{r}\left[n_{z i}\left(T_{e}, n_{e}\right)+\sum_{n^{\prime} l^{\prime}>n_{i}+1} n_{(z-1) i n^{\prime} l^{\prime}}\left(T_{e}, n_{e}\right)\right],
$$

where $A_{i j}^{r}$ is the transition probability for transition $i j, n_{z i}$ is the population of the excited level $i$ in the ion whose charge is $z$, and $n_{(z-1), i n^{\prime} l}$ is the population of the doubly excited level in the ion whose charge is $(z-1)$, with the same configuration as the level $i$, but with an additional electron in the level $n^{\prime} l^{\prime}$. As a number of authors have pointed out (see, for example, Walker (1972) and Gabriel and Jordan (1972)), if $n^{\prime} l^{\prime}$ is sufficiently large (in practice $>n_{i}+1$ ) the transitions $i n^{\prime} l^{\prime} \rightarrow j n^{\prime} l^{\prime}$ cannot be resolved from the transition $i \rightarrow j$, and therefore effectively increase the flux in the line $i \rightarrow j$. For $n^{\prime}<n_{i}+1$ the transition $i n^{\prime} l^{\prime} \rightarrow j n^{\prime} l^{\prime}$ can be resolved as a satellite line from $i \rightarrow j$, and in that case the emission is given by

$$
E_{Z z i j}=\frac{h c}{\lambda_{i j}} A_{i j n^{\prime} l^{\prime}}^{r}\left[n_{(z-1) i n^{\prime} l^{\prime}}\left(T_{e}, n_{e}\right)\right],
$$

where we have no longer made the assumption $A_{i j}^{r} \simeq A_{i j n^{\prime} l^{\prime}}^{r}$. 
Gabriel and Jordan (1972) have discussed the equation of statistical equilibrium which must be solved in order to calculate the state population functions. For most cases of interest, we may considerably simplify the equation of statistical equilibrium, and consequently, the excitation function can be expressed in simple form. For singly excited states, direct collisional excitation is the principal mechanism of population, and we may express the upper state population as

$$
n_{z i}=\frac{n_{e} n_{z g}\left(T_{e}\right) \alpha_{g i}^{\mathrm{ex}}\left(T_{e}\right)}{\Gamma_{i}^{r}}
$$

where $\Gamma_{i}^{r}$ is the total radiative width of the state $i\left[\right.$ i.e. $\left.\Gamma_{i}^{r}=\sum_{k} A_{i k}^{r}\right]$ and $\alpha_{g i}^{\mathrm{ex}}\left(T_{e}\right)$ is the collisional excitation rate from the ground state.

We may express the ground state density, $n_{z g}$, in terms of the relative population of the ionization stage of interest, $a_{z}{ }^{*}$, the relative abundance of the element, $A_{Z}$, and the number of hydrogen atoms per electron, $a_{\mathrm{H}}$. This latter quantity is approximately constant since the hydrogen and helium in the corona are completely ionized.

$$
n_{z g}=\left(n_{z g} / n_{Z}\right)\left(n_{Z} / n_{\mathrm{H}}\right)\left(n_{\mathrm{H}} / n_{e}\right) n_{e}=a_{z} A_{Z} a_{\mathrm{H}} n_{e} \cdot * *
$$

We shall also have occasion to use the fractional population of an excited state

$$
n_{z i}=\left(n_{z i} / n_{\mathrm{Z}}\right)\left(n_{\mathrm{Z}} / n_{\mathrm{H}}\right)\left(n_{\mathrm{H}} / n_{e}\right) n_{e}=f_{z i} A_{Z} a_{\mathrm{H}} n_{e}
$$

The emission function can be written as

$$
E_{Z z i j}^{\mathrm{ex}}=\frac{h c A_{i j}^{r} n_{e}^{2} a_{\mathrm{H}} A_{Z} a_{z}\left(T_{e}\right) \alpha_{g i}^{\mathrm{ex}}\left(T_{e}\right)}{\lambda_{i j}^{r}} .
$$

This equation is called the coronal excitation equation. We shall now discuss the modifications to Equation (5) which are necessary in order to discuss the line emission functions of ions for the hydrogen-like through neon-like isoelectronic sequences which are responsible for the coronal lines below $25 \AA$. We shall refer to the ions which have no metastable excited levels, and no levels with strong excitation rates from the ground level which have forbidden decays, as belonging to Class I. The coronal excitation equation will provide a good approximation in calculating the spectrum of these ions. Hydrogenic ions and lithium-like ions satisfy these criteria.

If an ion has a metastable excited level, this level will generally be strongly coupled to adjacent levels by radiative and collisional processes. It is then possible to solve the equation of statistical equilibrium for this group of strongly coupled levels separately. The population of other levels in the ion will still be governed by the coronal excitation equation. An example of this situation is provided by the metastable $1 s 2{ }^{3} S$ state in

* Jordan $(1969,1970)$ and Landini and Fossi (1972) have calculated $a_{z}$ for the abundant elements. ** This equation is not strictly valid if the ion contains metastable states which may have an appreciable steady state population. 
helium-like ions, which is strongly coupled with the $1 s 2 p^{3} P_{2,1,0}$ states. The relative intensities of the lines from these ions becomes density dependent at sufficiently high densities. The density dependent emission functions of the triplet states of helium-like ions has been discussed extensively in the literature (Gabriel and Jordan, 1972, 1973). We shall refer to ions with metastable excited levels as belonging to Class II.

In deriving Equation (5), we neglected population processes such as radiative recombination, and cascade from higher levels. The assumption that these processes are of minor importance is generally valid as long as the decay of the higher levels to the ground level is not forbidden. If such decays are forbidden, these levels will contribute to the population of the lowest lying excited levels by cascade processes, and Equation (5) will no longer describe the level populations. If there are no metastable states, we must solve a set on $N$ simultaneous equations, which involve population of each level by collisional excitation from the ground term, and by radiative decay from higher levels. The level population will then be expressed as a sum of terms such as appear in Equation (5), with coefficients which depend on the radiative decay rates from the levels responsible for the cascade. Walker et al. (1974c) show that the emission function may be written

$$
E_{i j}^{\mathrm{ex}}\left(T_{e}, n_{e}\right)=\frac{h c}{\lambda_{i j}} A_{i j}^{r} n_{e} n_{z, g} \frac{\sum_{k=i}^{N} \alpha_{g k}^{\mathrm{ex}}\left|\Gamma_{m}^{r} \delta_{m p}-A_{m p}^{r}\right|}{\prod_{k=i}^{N} \Gamma_{k}^{r}},
$$

where $m=i+1, \ldots N ; p=i, \ldots k-1, k+1, \ldots, N($ i.e., $p \neq k)$ and $A_{m p}^{r}=0$ for $p \geqslant m$. We have defined the total radiative width of the state $m, \Gamma_{m}^{r}=\sum_{k=1} A_{m k}^{r}$. The symbol $\delta_{m p}$ refers to the Kronecker delta function $\left[\delta_{m p}=1, m=p, \delta_{m p}=0 m \neq p\right]$.

The neon-like ion Fe XVII provides an example of an ion of this type. Figure 1 (taken from Walker et al., 1974c) shows the level structure of the excited levels of Fe XVII with principal quantum number 3 , and the principal decay paths of each level. We will refer to ions of this type as Class III.

A number of ions have metastable low lying excited states within the ground term, or within a low lying excited term. At sufficiently high temperatures and densities, the population in these low lying excited states can become substantial, and may exceed the population of the ground level. The coronal excitation equation must quite obviously be modified for these ions. Generally, the populations of the ground term, and of the low-lying excited states may be computed by regarding these levels as strongly coupled, and solving the statistical equilibrium equation ignoring all higher levels. Once the populations of these metastable levels have been determined, we can calculate the population of excited states by applying Equation (5) to each highly populated level. If $f_{g}, f_{g^{\prime}}, f_{g^{\prime \prime}}, \ldots$ are the fractional populations of these levels then we may write the emission function of a line as

$$
E_{Z z i j}^{\mathrm{ex}}=\frac{h c A_{i j}^{r} n_{e}^{2} a_{\mathrm{H}} A_{Z} a_{z}\left(T_{e}\right) \sum f_{z g} \alpha_{g i}^{\mathrm{ex}}\left(T_{e}\right)}{i_{i j}} .
$$




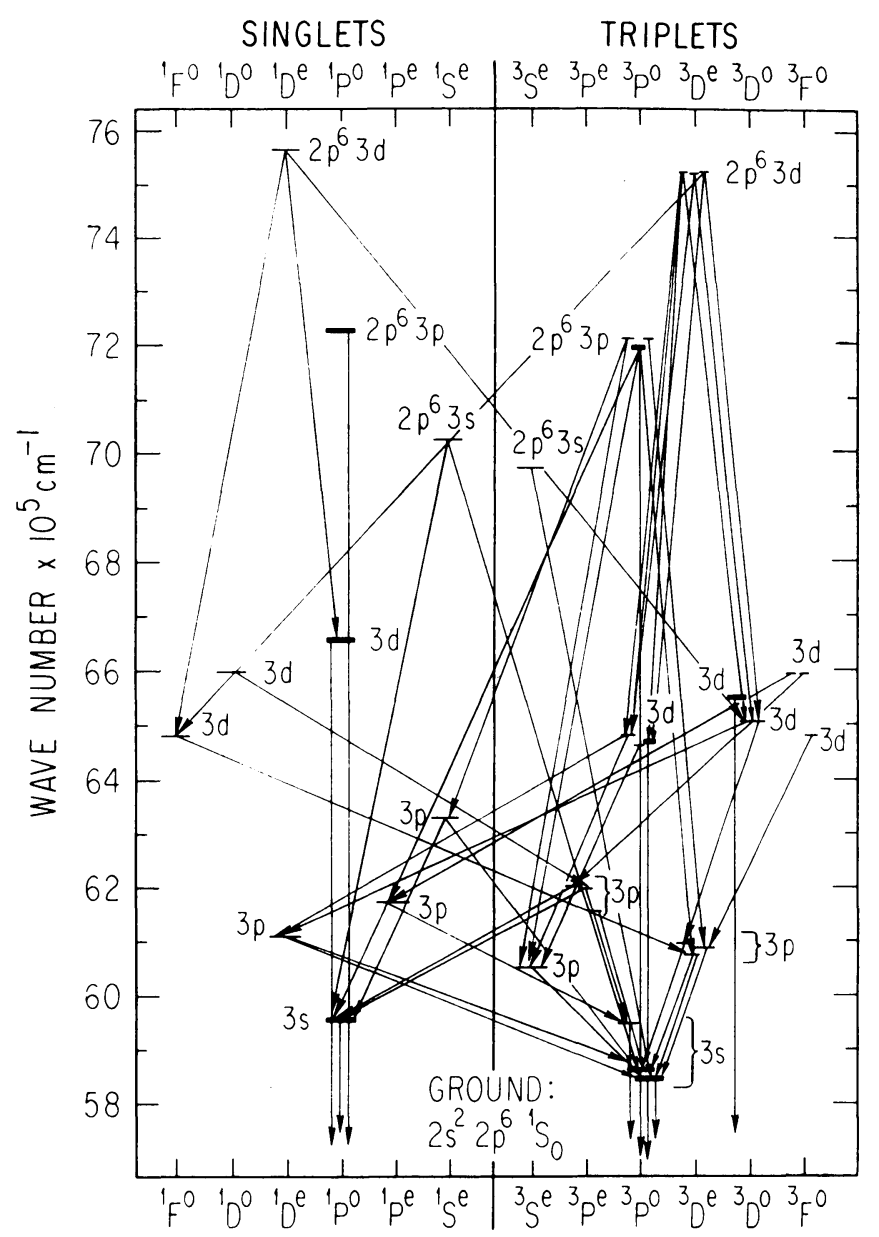

Fig. 1. Level diagram for Fe XVII, showing the principal decay paths for the first 36 excited levels. Those levels with transitions to the ground level are shown in bold type.

Examples of ions with metastable low lying excited states are those of the berylliumlike and boron-like isoelectronic sequences. The density dependent populations of the $2 S^{2}{ }^{1} S$ ground state and $2 s 2 p^{3} P_{2,1,0}$ excited states of beryllium-like ions have been extensively discussed by Jordan (1971) and Munro et al. (1971). We will refer to ions with metastable low lying excited states as Class IV. In Table I, we have classified the ions of the ten simplest isoelectronic sequences.

The collisional excitation function which appears in Equation (5) is generally expressed in terms of a collision strength, $\Omega_{g i}$, which is defined as

$$
\sigma=\frac{\pi a_{0}^{2} \Omega_{g i} I_{\mathrm{H}}}{\left(2 J_{g}+1\right) E},
$$

where $\sigma$ is the excitation cross section, $a_{0}$ the Bohr radius, $I_{\mathrm{H}}$ the rydberg, $J_{g}$ the spin of 
the initial state, and $E$ the incident electron energy. For a Maxwellian distribution, the excitation rate is

$$
\alpha_{g i}^{\mathrm{ex}}=\frac{2 \sqrt{2}}{\left(2 J_{g}+1\right)} \frac{\pi a_{0}^{2}}{\sqrt{m \pi k T}} I_{\mathrm{H}} \int_{E_{i}}^{x} \Omega_{g \mathrm{i}} \exp (-E / k T) \frac{\mathrm{d} E}{k T} .
$$

$E_{i}$ is the threshold energy of the excitation under consideration.

\section{TABLE I}

Classification of the $\mathrm{H}$ - Ne-like Ions

\begin{tabular}{|c|c|c|}
\hline Sequence & Class & Comments \\
\hline $\mathrm{H}_{\mathrm{I}}$ & I & \\
\hline $\mathrm{He} \mathrm{I}$ & II, III & metastable level $1 s 2 s{ }^{3} S ; 1 s n^{3} L$ cascade to $1 s 2{ }^{3} L$ \\
\hline $\mathrm{Li}$ I & I & \\
\hline $\mathrm{Be}_{\mathrm{I}}$ & IV & metastable levels $2 s 2 p^{3} P_{2,1,0}$ \\
\hline B I & III, IV & $\begin{array}{l}\text { ground term contains } 2 p^{2} P_{3 / 2}, 1 / 23 p^{2} P \text { levels decay by } \\
\text { cascade to } 3 s^{2} S\end{array}$ \\
\hline $\mathrm{C}$ I & III, IV & $\begin{array}{l}\text { ground term contains } 2 p^{2}{ }^{3} P_{2,1,0}, 2 p 3 p \text { levels decay by } \\
\text { cascade to } 2 p 3 s\end{array}$ \\
\hline $\mathrm{N}_{\mathrm{I}}$ & III, IV & $\begin{array}{l}\text { ground configuration contains } 2 p^{3}{ }^{4} S_{3 / 2} \text { and } \\
2 p^{3}{ }^{2} D_{5 / 2,3 / 2} ; 2 p^{2} 3 p \text { levels decay by cascade to } 2 p^{2} 3 s\end{array}$ \\
\hline $\mathrm{O}_{\mathrm{I}}$ & III, IV & $\begin{array}{l}\text { ground configuration contains } 2 p^{4}{ }^{3} P_{2,1}, 0,2 p^{4}{ }^{1} D \text { and } \\
2 p^{4} S ; 2 p^{3} 3 p \text { levels decay by cascade to } 2 p^{3} 3 s\end{array}$ \\
\hline$F_{1}$ & III, IV & $\begin{array}{l}\text { ground term contains } 2 p^{5}{ }^{2} P_{1 / 2,3 / 2}, 2 p^{4} 3 p \text { levels decay by } \\
\text { cascade to } 2 p^{4} 3 s \text { levels }\end{array}$ \\
\hline $\mathrm{Ne}$ I & III & $\begin{array}{l}2 p^{5} 3 p \text { levels and } 2 s 2 p^{6} 3 / \text { levels decay by cascade to } 2 p^{5} 3 s \\
\text { and } 2 s^{2} 2 p^{5} 3 / \text { levels respectively. }\end{array}$ \\
\hline
\end{tabular}

The collision strength can be expressed in terms of the oscillator strength, $f$, and the gaunt factor $g$, using the modified Bethe approximation of Van Regemorter (1962).

$$
\Omega_{g i}=\frac{8 \pi I_{\mathrm{H}}}{\sqrt{ } 3 E} f_{i} g_{i} .
$$

For two excitations which are similar, we may presume that the gaunt factors are equal, and derive the useful relation

$$
\Omega_{g i}\left(E_{i}\right)=\frac{f_{i} E_{i^{\prime}}}{f_{i^{\prime}} E_{i}} \Omega_{g i^{\prime}}\left(\begin{array}{l}
E_{i^{\prime}} \\
E_{i}
\end{array}\right) .
$$

In the succeeding subsections, we will briefly discuss the determination of the atomic rate parameter required to evaluate Equations (5), (7), (8), and (10). Emphasis will be placed on the calculation of collisional excitation cross sections. References for oscillator strengths and transition probabilities are given by Gabriel and Jordan (1972) and Walker (1972). 


\section{(i) Hydrogenic Ions}

Burgess et al. (1970) have calculated the collisional excitation cross sections for the $1 s-2 s$ and $1 s-2 p s$ excitations in hydrogenic ions, using the Coulomb Born II approximation.

Walker (1972) has tabulated the temperature averaged collision strength $z^{2} \Omega$ for these transitions

$$
\overline{z^{2} \Omega}=\frac{1}{2 J_{g}+1} \int_{1}^{\infty} \mathrm{d} \varepsilon\left\{z^{2} \Omega \exp [-(\varepsilon-1) / \theta]\right\} / \theta
$$

(where $\varepsilon=E / E_{i}$ and $\theta=k T / E_{i}$ ) using the collision strengths given by Burgess et al. We may obtain the collision strength for higher excitations $(1 s-n s$ and $1 s-n p)$ by using Equation (10). Recently, Walker (1974) has calculated the cross section for $1 s-2 s$ and $1 s-2 p$ excitation using relativistic wave functions. He finds substantial corrections to the results of Burgess $\epsilon t$ al., for large $Z$, however, for values of $Z$ of importance for coronal studies, $(Z \leqslant 28)$ the corrections are small.

(ii) Helium-like Ions

Until quite recently, there were few calculations available for the collision strengths of excitations in helium-like ions. Collision strengths were derived from the hydrogenic calculations of Burgess et al. for the singlet $\left({ }^{1} S\right.$ and $\left.{ }^{1} P\right)$ excitations using Equation (10) by Walker (1972), and by Gabriel and Jordan (1972) using the $R$ matrices tabulated by Burgess et al. Gabriel and Jordan were able to derive collision strengths for excitation of both the singlet $\left({ }^{1} P\right.$ and $\left.{ }^{1} S\right)$ and triplet $\left({ }^{3} P\right.$ and $\left.{ }^{3} S\right)$ lines. Tully (1974) has recently calculated the excitation cross section for the 1 sns ${ }^{1} S(n=2, \ldots 6)$ and 1 snp ${ }^{1} P(n=2, \ldots 6)$ levels in the Coulomb Born Approximation. The cross section obtained by this more accurate technique are equal, within a few percent, to the cross section derived by Walker (1972), using Equation (10), from the hydrogenic cross sections, as shown by Table II.

\section{TABLE II}

Comparison of $\mathrm{O}$ VII collision strengths

\begin{tabular}{|c|c|c|c|c|}
\hline \multicolumn{2}{|c|}{ Energy (in units of threshold energy) } & 1 & 2 & 3 \\
\hline \multirow[t]{2}{*}[(f\lambda)_{\text{ovinis_-2p}}/(f\lambda)_{\text{oviril}\alpha}]{} & $z^{2} \Omega^{\mathrm{CBO}}\left(1^{2} S-2^{2} P\right)^{\mathrm{a}}$ & 2.765 & 4.171 & 5.444 \\
\hline & $z^{2} \Omega^{\mathrm{CB}}\left(1^{1} S-2^{1} P\right)^{\mathrm{b}}$ & 2.825 & 4.675 & 5.975 \\
\hline \multirow{2}{*}{ 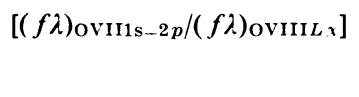 } & $z^{2} \Omega^{\mathrm{CBO}}\left(1^{2} S-2{ }^{2} S\right)^{\mathrm{a}}$ & 0.676 & 0.718 & 0.741 \\
\hline & $z^{2} \Omega^{\mathrm{CB}}\left(1^{1} S-2^{1} P\right)^{\mathrm{b}}$ & 0.803 & 0.868 & 0.880 \\
\hline \multirow[t]{2}{*}{$(f \lambda)_{\mathrm{OVIIIs}-3 p} /(f \lambda)_{\left.\mathrm{OVIII} L_{\lambda}\right]}$} & $z^{2} \Omega^{\mathrm{CBO}}\left(1^{2} S-2^{2} P\right)^{\mathrm{a}}$ & 0.508 & 0.767 & 1.000 \\
\hline & $z^{2} \Omega^{\mathrm{CB}}\left(1^{1} S-3^{1} P\right)^{\mathrm{b}}$ & 0.615 & 0.996 & 1.256 \\
\hline
\end{tabular}

a Tully (1974).

b Burgess et al. (1970). 
However, it is recommended that the more accurate cross section of Tully be adopted for future work. For the triplet levels, the cross sections given by Gabriel and Jordan are the most reliable available.

\section{(iii) Lithium-like Ions}

Gabriel and Jordan (1972) and Sampson and Parks (1974) have given extensive accounts of theoretical and experimental studies of the cross sections of the lithium-like ions.

Bely (1966a, 1966b) and Bely and Petrini (1970) and Flower and Launay (1972) have calculated threshold collision strengths for the transitions $2 s-n l$ and $2 p-n l$ in lithium-like ions.

The calculations of Bely and Bely and Petrini appear to result in good agreement with observation, except for the $2 s-3 p$ excitation, where the cross sections of Burke et al. (1966) appear to result in closer agreement. Heroux and Cohen (1971), Heroux et al. (1972), and Malenovsky and Heroux (1973) have analyzed the relative intensities of the lines of the lithium-like ions $\mathrm{O}$ VI, $\mathrm{Ne}$ viII, and $\mathrm{Mg} \mathrm{x}$. They find that their observed intensities are consistent with the theoretical cross sections.

\section{(iv) Beryllium-Like Ions}

Jordan (1971) and Munro et al. (1971) have discussed the density dependent population of the $2 s^{2}{ }^{1} S$ and $2 s 2 p{ }^{3} P$ levels of beryllium-like ions, and have computed the stacistical equilibrium of these levels as a function of density for $\mathrm{C}$ III, $\mathrm{N} \mathrm{IV}, \mathrm{O} v$, and $\mathrm{Si} \mathrm{XI}$. Once the populations of the ground level, and of the three metastable levels have been calculated, the modified coronal excitation equation (Equation (7)) can be used to calculate the populations of the higher excited levels. Gabriel and Jordan (1972) and Sampson and Parks (1974) have discussed the theoretical and experimental studies of collision strengths for the beryllium-like ions. The most extensive theoretical calculations are those of Eissner which are unpublished. However, Gabriel and Jordan quote a number of Eissner's results, and compare then with the experimental observations of excited rates by Tondello and McWhirter (1971) and Johnson and Kunze (1971) for Ne VII and Si XI. The agreement is fair for most transitions, however, there are substantial differences for some transitions. In view of the difficulty of interpreting the experimental observations, which require the determination of the population of the $2 s 2{ }^{3} P$ metastable levels in a transient laboratory plasma, the theoretical cross sections, where available, are likely to be more reliable.

\section{(v) Neon-Like Ions}

Loulergue and Nussbaumer (1973) and Walker et al. (1974c) have recently discussed the coronal spectrum of Fe XVII. Because the $2 s^{2} 2 p^{5} 3 s$ levels are populated primarily by cascade, the collisional excitation rates and radiative decay rates for the first 36 excited levels must be known before the relative and absolute intensities of the principal lines of Fe XVII, which lie between $\sim 12$ and $\sim 17 \AA$, can be calculated. Figure 1 illustrates the complexity of the spectrum of Fe XVII. However, Loulergue (1971, 1973) and Garstang (1969) have calculated all of the required transition rates, and Bely and 


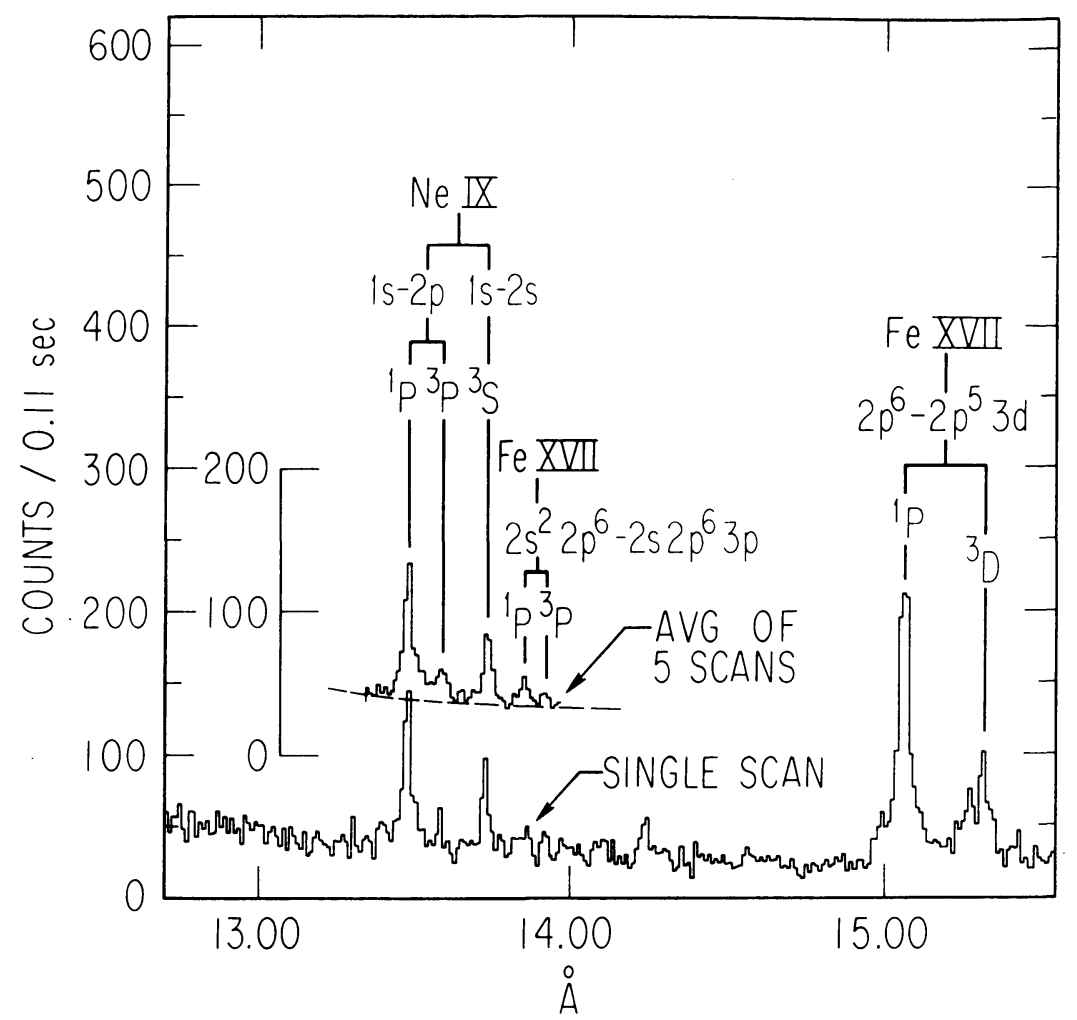

Fig. 2. Portion of the spectrum recorded by the KAP spectrometer on O VI-17 for 1969, March 20, showing the Fe. XVII $2 s^{2} 2 p^{6}{ }^{1} S-2 s 2 p^{6} 3 p^{1} P$ and ${ }^{3} P$ lines.

Bely (1967) and Flower (1971) have calculated threshold collision strengths for the transitions $2 p-3 s, 3 p$, and $3 d$. For the $2 s-3 s, p, d$ singlet excitations, Bely and Bely have estimated collision strengths by using cross sections for lithium-like ions. Using these atomic rate constants, Loulergue and Nussbaumer have calculated the relative intensity of the eight lines observed in the coronal spectrum from $2 p^{5} 3 s, 2 s 2 p^{6} 3 p$, and $2 p^{5} 3 d$ configurations of Fe XVII. In table III these results (column la) are compared with the line intensities observed by Walker et al. (1974c) and Parkinson (1975). The observations of Walker et al. are shown in Figures 2 and 3. Loulergue and Nussbaumer have estimated the cross sections for the $2 s 2 p^{6} 3 s, 3 p$ and $3 d$ triplet excitations by assuming that the collision strengths are equal to the singlet collision strengths multiplied by the statistical weights of the states. The relative intensities derived with this assumption are given in column $1 \mathrm{~b}$ of Table III. Walker et al. (1974c) have made somewhat different assumptions regarding the $2 s-3 s, 3 p$ and $3 d$ excitation rates. They made use of the collision strengths for beryllium-like ions calculated by Eissner to derive the $2 s-3 s$ and $2 s-3 d$ singlet excitation rates, and used the observed intensities of the $2 s 2 p^{6} 3 p$ singlet and triplet lines, and the intensities of the $2 s^{2} 2 p^{5} 3 s^{1} P$ and ${ }^{3} P$ lines to derive collision strengths for the $2 s 2 p^{6} 3 s^{3} S$ and $2 s 2 p^{6} 3 d^{3} D$ levels. 
TABLE III

Comparison of experimental and theoretical line intensities in Fe xvII

Transition Theory

\section{Experiment}

$(\AA)$

(1a) (1b)

(2)

4 Jan. 1967 (2)

20 Mar. $1969(2)$

30 Nov. $1971(3)$

$\begin{array}{lllllllll}3 s-2 p & 17.10 & 0.23 & 0.74 & 0.59 & 1.25 & 0.75 & 0.73 \\ 3 s-2 p & 17.05 & 0.58 & 0.97 & 0.91 & 1.50 & 0.80 & 0.90 \\ 3 s-2 p & 16.77 & 0.38 & 0.54 & 0.51 & 0.70 & 0.72 & 0.65 \\ 3 d-2 p & 15.45 & 0.008 & 0.08 & 0.09 & 0.05 & 0.09 & 0.11 \\ 3 d-2 p & 15.26 & 0.33 & 0.37 & 0.37 & 0.43 & 0.56 & 0.48 \\ 3 d-2 p & 15.01 & 1.00 & 1.00 & 1.00 & 1.00 & 1.00 & 1.00 \\ 3 p-2 s & 13.88 & 0.002 & 0.10 & 0.03 & - & 0.03 & 0.04 \\ 3 p-2 s & 13.82 & 0.15 & 0.15 & 0.07 & - & 0.07 & 0.07 \\ 4 d-2 p & 12.26 & - & - & - & 0.07 & - & 0.08 \\ 4 d-2 p & 12.12 & - & - & - & 0.04 & - & 0.09\end{array}$

(1a) Loulergue and Nussbaumer $\left(T=5.5 \times 10^{6}\right)\left[\Omega_{2 s^{2} 2 p^{6}{ }^{1} S_{-}-2 s 2 p 3 l}{ }^{3} L \equiv 0\right]$

(1b) Loulergue and Nussbaumer $\left(T=5.5 \times 10^{6}\right)$

(2) Walker, Rugge, and Weiss $\left(T-4.0 \times 10^{6}\right)$

(3) Parkinson

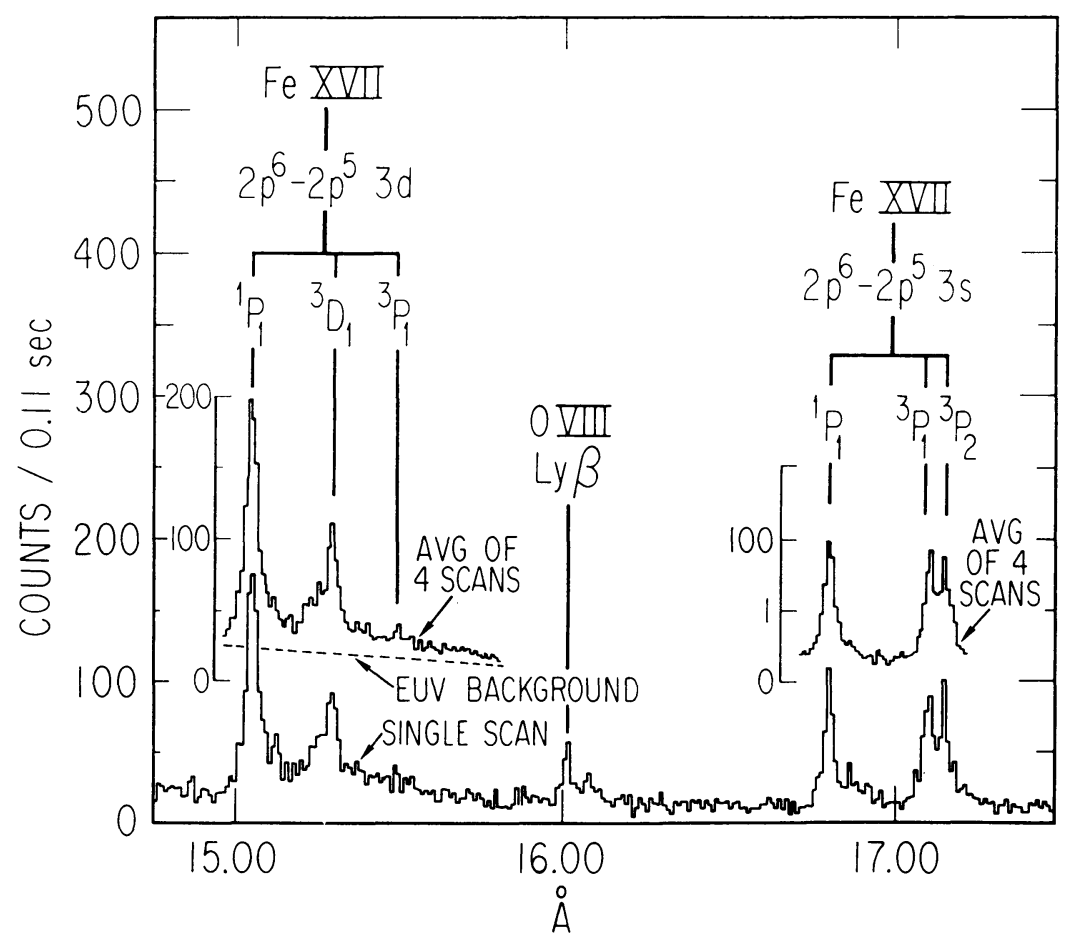

Fig. 3. Portion of the spectrum recorded by the KAP spectrometer on O VI-17 for 1969, March 20 , showing the Fe xvII $2 s^{2} 2 p^{6}{ }^{1} S \quad 2 s^{2} 2 p^{5} 3 d^{1} P_{.}{ }^{3} D$, and ${ }^{3} P$, and $2 s^{2} 2 p^{61} S_{0} \quad 2 s^{2} 2 p^{5} 3 s^{1} P_{1},{ }^{3} P_{1}$ and ${ }^{3} P_{2}$ lines. 
TABLE IV

Threshold collision strengths for Fe XVII

\begin{tabular}{|c|c|c|}
\hline Configuration & Level & Collision strength $\left(\times 10^{3}\right)$ \\
\hline
\end{tabular}

$\begin{array}{rrlr}2 s 3 p^{6} 3 d & { }^{1} D_{2} & 65.2 & 73 \\ & { }^{3} D_{1} & - & 100 \\ 2 s 2 p^{6} 3 p & { }^{1} P_{1} & 22.4 & 10.1 \\ & { }^{3} P_{1} & - & 6.5 \\ 2 s 2 p^{6} 3 s & { }^{1} S_{0} & 32.4 & 30.1 \\ & { }^{3} S_{1} & - & 45\end{array}$

TABLE V

Parentage of Fe XVII lines observed in the corona

\begin{tabular}{|c|c|c|c|c|c|c|c|c|c|c|c|}
\hline \multirow[t]{2}{*}{ Level } & \multicolumn{2}{|c|}{$2 s^{2} 2 p^{5} 3 s$} & \multicolumn{2}{|c|}{$2 s^{2} 2 p^{5} 3 p$} & \multirow[b]{2}{*}{${ }^{3} D_{1}$} & \multirow[b]{2}{*}{${ }^{1} D_{2}$} & \multirow[b]{2}{*}{${ }^{3} D_{0}$} & \multirow[b]{2}{*}{${ }^{1} P_{1}$} & \multirow[b]{2}{*}{${ }^{3} P_{1}$} & \multirow[b]{2}{*}{${ }^{1} P_{2}$} & \multirow[b]{2}{*}{${ }^{1} S_{0}$} \\
\hline & ${ }^{3} P_{1}$ & ${ }^{1} P_{1}$ & ${ }^{3} S_{1}$ & ${ }^{3} D_{2}$ & & & & & & & \\
\hline $2 p^{5} 3 s^{3} P_{2}$ & - & - & 0.949 & 0.390 & 0.048 & 0.446 & - & - & 0.068 & 0.007 & - \\
\hline $2 p^{5} 3 s^{3} P_{1}$ & 1.00 & - & 0.051 & 0.610 & 0.952 & 0.549 & 0.892 & 0.451 & 0.600 & 0.015 & 0.500 \\
\hline $2 p^{5} 3 s^{3} P_{0}$ & - & 1.00 & - & - & - & 0.005 & 0.108 & 0.549 & 0.332 & 0.978 & 0.500 \\
\hline $2 p^{5} 3 d^{3} P_{1}$ & - & - & - & - & - & - & - & - & - & - & - \\
\hline $2 p^{5} 3 d^{3} D_{1}$ & - & - & - & - & - & - & - & - & - & - & - \\
\hline $2 p^{5} 3 d^{1} P_{1}$ & - & - & - & - & - & - & - & - & - & - & - \\
\hline
\end{tabular}

Level $2 s^{2} 2 p^{5} 3 d$

${ }^{3} P_{0} \quad{ }^{3} P_{1} \quad{ }^{1} F_{3} \quad{ }^{3} P_{2} \quad{ }^{3} D_{1} \quad{ }^{3} F_{2} \quad{ }^{1} D_{2} \quad{ }^{1} P_{1}$

$2 p^{5} 3 s^{3} P_{2}$

$\begin{array}{llllllll}0.947 & 0.097 & 0.424 & 0.621 & - & 0.007 & 0.106 & - \\ 0.048 & 0.022 & 0.576 & 0.370 & 0.003 & 0.423 & 0.503 & - \\ 0.005 & - & 0.0005 & 0.009 & - & 0.570 & 0.391 & - \\ - & 0.881 & - & - & - & - & - & - \\ - & - & - & - & 0.997 & - & - & - \\ - & - & - & - & - & - & - & 0.999\end{array}$

$2 p^{5} 3 s^{3} P_{1}$

$2 p^{5} 3 d^{3} P_{1}$

$2 p^{5} 3 d^{3} D_{1}$

$2 p^{5} 3 d^{1} P_{1}$

\begin{tabular}{lllllll}
$2 s 2 p^{6} 3 s$ & \multicolumn{3}{c}{$2 s 2 p^{6} 3 p$} & \multicolumn{3}{c}{$2 s 2 p^{6} 3 d$} \\
\hline${ }^{3} S_{1}$ & ${ }^{1} S_{0}$ & ${ }^{3} P_{1}$ & ${ }^{1} P_{1}$ & ${ }^{3} D_{1}$ & ${ }^{1} D_{2}$ \\
\hline & & & & & \\
0.594 & - & 0.186 & 0.010 & 0.266 & 0.204 \\
0.287 & 0.598 & 0.139 & 0.021 & 0.316 & 0.369 \\
0.119 & 0.402 & 0.054 & 0.010 & 0.074 & 0.149 \\
- & - & - & - & 0.197 & - \\
- & - & - & - & 0.119 & 0.073 \\
- & - & - & - & 0.013 & 0.154 \\
- & - & 0.621 & - & 0.015 & 0.005 \\
- & - & - & 0.959 & - & 0.046
\end{tabular}


The relative intensities derived by Walker et al. are compared with the observations in Table III. The collision strengths derived by Walker et al. are compared with those derived by Bely and Bely in Table IV. The relative contribution of each level to the flux observed in each of the lines of Fe XVII as calculated by Walker et al. is shown in Table V. The arrays tabulated in Table $\mathrm{V}$ are the products

$$
\frac{A_{i j}^{r}\left|\Gamma_{m}^{r} \delta_{m p}-A_{m p}^{r}\right|}{\prod_{k=i}^{N} \Gamma_{k}^{r}}
$$

required for the evaluation of Equation (6).

\section{(vi) Calculation of the Coronal Line Spectrum}

Detailed studies of the excitation of ions of the boron-like* through flourine-like sequences have not yet been carried out. However, a number of authors (Tucker and Koren, 1971; Mewe, 1972; and Landini and Fossi, 1970) have calculated the coronal line spectrum below $100 \AA$. The calculations of Tucker and Koren are the most complete. For ions of the beryllium-like through the fluorine-like isoelectronic sequences, Tucker and Koren have scaled the collision strengths of Bely and Petrini (1970) for the $2 p-n l$ excitations in lithium-like ions, using Equation (10). Mewe, on the other hand, has derived effective gaunt factors for a large number of transitions, using the best available theoretical and experimental data for each isoelectronic sequence. Neither author includes the effects of cascades. The general character of the coronal spectrum is shown in Figure 4, which is based on extensive unpublished calculations by the author, in collaboration with Mrs. Kay Weiss at the Aerospace Corporation. Because of the requirement for accurate calculations of the coronal X-ray spectrum in order to interpret the X-ray filtergrams obtained by the S-054 and S-056 experiments on Skylab, extensive calculations of the coronal line spectrum are in progress at American Science and Engineering by Drs Vaiana and Kreiger and their associates, and at the Aerospace Corporation by Drs McKenzie and Walker. The results of these calculations should be available within a year of the date of the present Symposium.

\section{(vii) Dielectronic Recombination Lines and Satellite Lines}

The major process responsible for the population of doubly excited levels in thermal equilibrium is dielectronic recombination (Gabriel and Paget, 1972) for most isoelectronic sequences. However, for certain isoelectronic sequences, such as neon-like ions (Walker et al., 1974c) inner shell excitation can be an important process. For those cases where dielectronic recombination dominates

$$
E_{Z z i j}^{\mathrm{d}}=\frac{h c}{i_{i j}} A_{i j}^{r} n_{e}^{2} a_{\mathrm{H}} A_{Z} a_{z}\left(T_{e}\right) \sum_{n^{\prime} l^{\prime}>n_{i}+1} \frac{\alpha_{g i n^{\prime} l^{\prime}}^{\mathrm{di}}\left(T_{e}\right)}{\Gamma_{i n^{\prime} l^{\prime}}^{r}+\Gamma_{i n^{\prime} l^{\prime}}^{\mathrm{a}}},
$$

* Widing (1966) has studied the excitation of some lines of the boron-like ion Si x. 

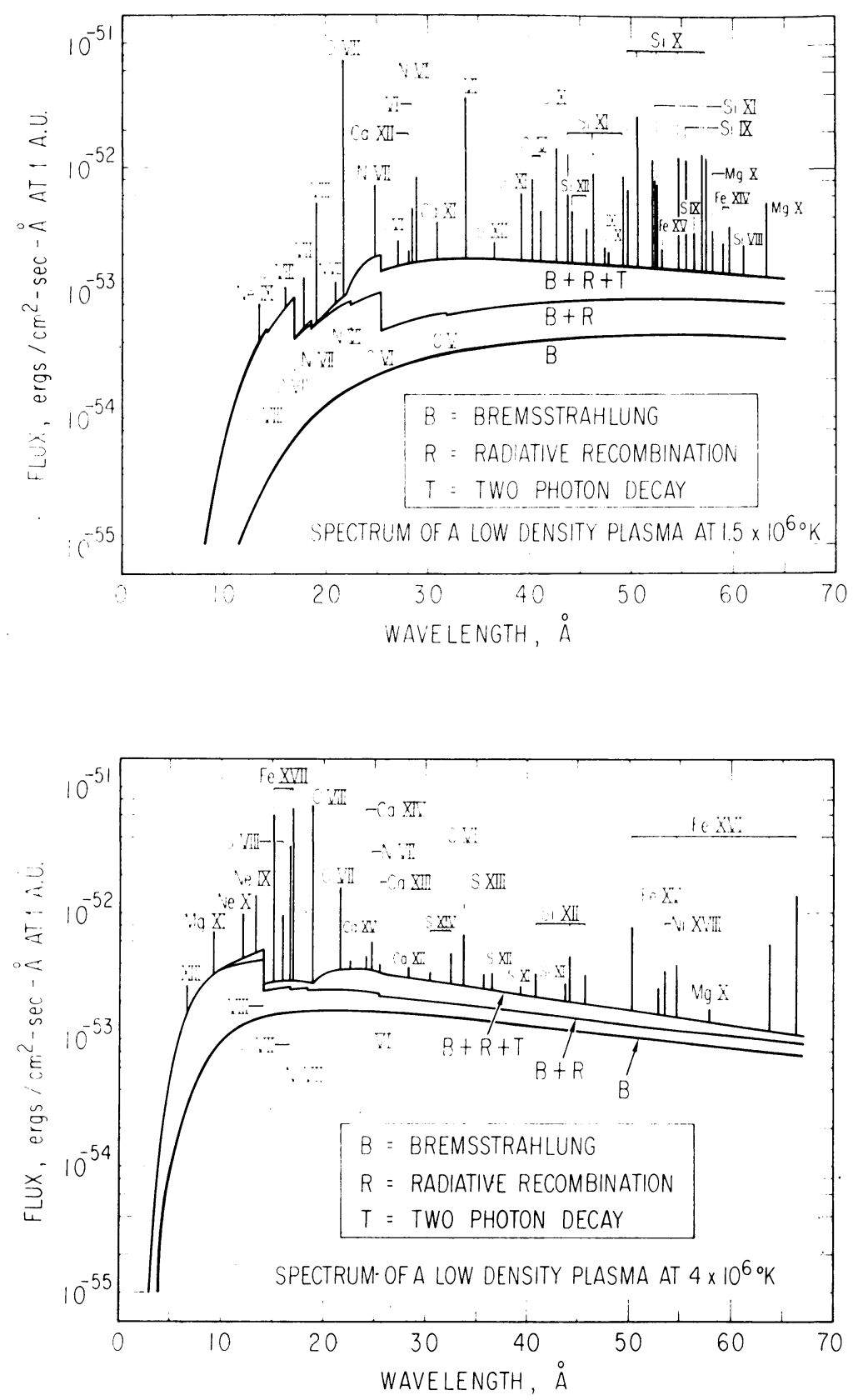

Fig. 4. Coronal Spectrum for $1.5 \times 10^{6} \mathrm{~K}$ and $4.0 \times 10^{6} \mathrm{~K}$. Only allowed transitions have been shown in order to avoid confusion, so that a number of strong lines (such as the helium-like forbidden lines) are not shown. Note the importance of two photon decay for the continuum at $1.5 \times 10^{6} \mathrm{~K}$. 
where $\alpha_{\mathrm{gin} \mathbf{n}^{\prime} l^{\prime}}^{\mathrm{di}}$ is the dielectronic recombination rate for the formation of the state $i n^{\prime} l^{\prime}$ in ionization stage $(z-1)$ from the ground state of ionization stage $z$, and $\Gamma_{\mathrm{in}{ }^{\prime}}^{\mathrm{a}}$, is the autoionizing width of the state. The same equation without the summation is the emission function of an individual satellite line. The lower limit of the summation in equation 12 will depend on the resolution of the spectrometer making the observations. Since most calculations of the total dielectronic recombination rate have assumed that $n^{\prime} l^{\prime}$ is large, the summation $\alpha^{\mathrm{di}}$ in Equation (12) may be taken equal to the total dielectronic recombination rate. Shore (1969) has carried out the most accurate computation of this rate for low $Z$ ions.

For highly charged helium-like ions the radiative width for states with $n^{\prime} \sim n_{i}$ becomes comparable to the autoionizing width, and the satellites with $n^{\prime}=n_{i}+1$, and $n^{\prime}=n_{i}$ become prominent spectral features (Walker and Rugge, 1971), approaching the intensity of the resonance line for Fe Xxv (Grineva et al., 1973). Tucker and Koren (1971) and Walker (1972) have discussed the evaluation of the emission function in Equation (12). Gabriel (1972) has given a definitive account of the wavelengths and intensities of the important satellites to the lines of the helium-like ions which arise from the $1 s 2 s 2 p$ and $1 s 2 p^{2}$ configurations of the lithium-like ion.

\section{(viii) The $X$-Ray Continuum}

Three processes make substantial contributions to the coronal X-ray continuum, radiative recombination, bremsstrahlung, and two photon decay of the metastable $2 s^{2} S$ and $1 s 2 s^{1} S$ levels of hydrogenic and helium-like ions. Culhane (1969) has given a complete account of the calculation of the spectrum for the first two processes. Walker (1972) and Tucker and Koren (1971) have discussed the calculation of the spectrum for two photon decay of hydrogenic and helium-like ions. Walker et al. (1974a) have tabulated the fractional contribution of various elements, and processes for several wavelengths and temperatures. The relative importance of the various continuum processes is shown in Figure 4. Figure 4 emphasizes the importance of two photon decay at temperatures between $\sim 1 \times 10^{6} \mathrm{~K}$ and $4 \times 10^{6} \mathrm{~K}$, and at wavelengths $\lambda>20 \AA$. The hydrogenic and helium-like ions of oxygen and carbon are chiefly responsible for this flux.

Walker et al. (1974a, b) have made detailed comparisons of the observed X-ray continuum, and the continuum calculated for a coronal model derived from line fluxes. Figure 5 (from Walker et al., 1974b) compares the experimental and theoretical continuum between 8 and $25 \AA$. The agreement is good between 8 and $14 \AA$, however, above $15 \AA$, the observed continuum is too large by a factor of $\sim 2$. Walker et al., suggest that the 'excess' continuum is due to weak unresolved lines from ions such as Fe XVIII $(\sim 16 \AA), \quad$ Cr XV-Cr XI $(\sim 16-22 \AA), \quad$ Ca XVI-Ca XI $(\sim 17-25 \AA)$, and Ar XV-Ar XVI $(\sim 23-25 \AA)$.

\section{(b) DIAGNOSTIC TECHNIQUES FOR THE CORONAL PLASMA}

A number of techniques have been developed which, in principle, allow the temperature and density structure of the coronal plasma to be determined without the development 
of a detailed model. These techniques depend on line intensity ratios which are sensitive to a particular parameter such as temperature or density. However, as we shall see, some care must be exercised in interpreting such intensity ratios in terms of isothermal models.

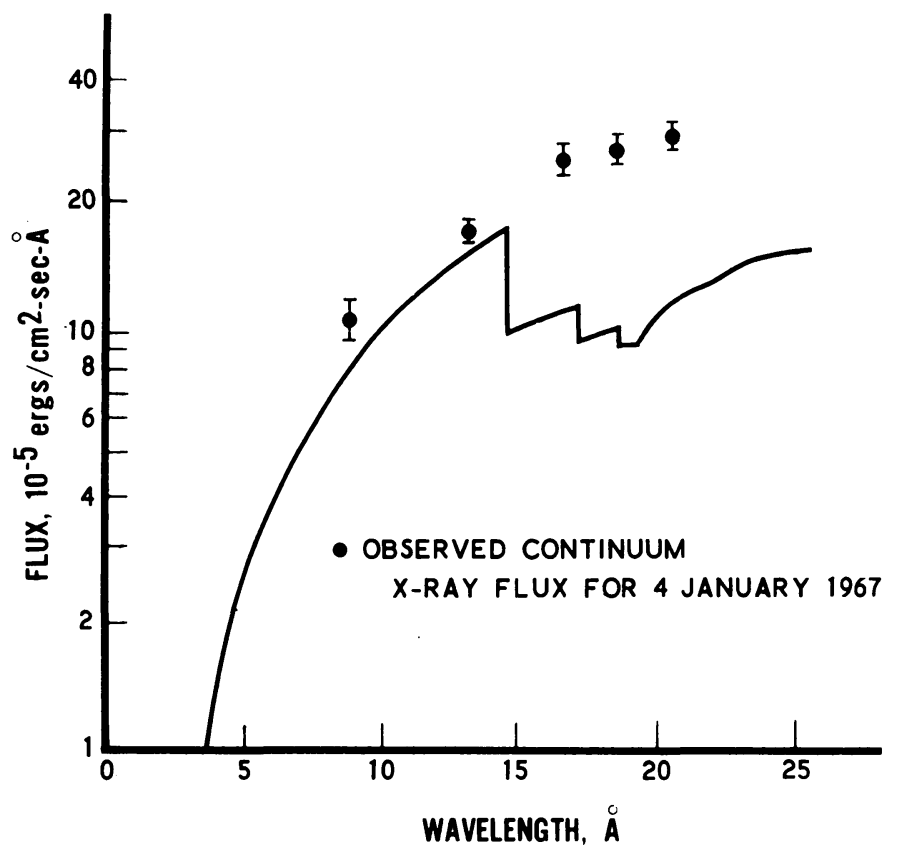

Fig. 5. Continuum fluxes observed on the $\mathrm{O}$ vi-10 satellite compared with the fluxes calculated from a coronal model derived from observed line fluxes. The model used is the one shown in Figure 7.

\section{(i) Density}

The dependence of line intensities on density comes about in two ways:

(1) Metastable excited levels may be competitively de-excited by radiative decay or collisional transfer to an adjacent level which has an allowed decay.

(2) Some ions have compound ground states whose upper level(s) are metastable, or low-lying metastable excited states. These metastable states become populated at sufficiently high densities and the intensities of lines which are preferentially excited from these metastable levels thus become strongly density dependent.

An example of the first situation is the intensity of the $1 s^{2}{ }^{1} S-1 s 2{ }^{3} S$ transition in helium-like ions. Gabriel and Jordan $(1972,1973)$ have analyzed the density dependence of this line in detail. A number of authors (Rugge and Walker, 1970, 1971; Acton et al., 1972, Bonnelle et al., 1973) have studied the relative intensity of the forbidden $\left(1 s^{2}{ }^{1} S-1 s 2 s^{3} S\right)$ and intercombination $\left(1 s^{2}{ }^{1} S-1 s 2 p^{3} P\right)$ lines from $\mathrm{O}$ vII, $\mathrm{Ne} I \mathrm{X}$ and $\mathrm{Mg}$ XI in the corona. However, only upper limits to the coronal density have been derived from these observations. There are a number of examples of the latter situation. Some ions which have been studied in the case of the solar corona are sum- 
marized in Table VI. The line intensity ratios tabulated are sensitive over the range of densities from $10^{4}-\sim 10^{14}$.

The $\Delta n=1$ transitions in beryllium-like ions will share the same density dependent behavior as the $\Delta n=0$ transition listed in Table VI. For example, the upper level of the $2 p^{2}{ }^{3} P-2 p 3 d^{3} D$ line will be populated primarily by excitation from the $2 s 2 p^{3} P$ level. The study of the $\Delta n=1$ transitions offers certain advantages in comparison with the $\Delta n=0$ transitions. Line multiplets which have their wavelengths very close together, may be selected thereby reducing the problem of instrument calibration. Furthermore, since the dependence of collisional excitation rates on temperature goes mainly as $\exp (-\Delta E / k T)$, where $\Delta E$ is the excitation energy of the upper level, lines which are close together in wavelength will generally have excitation functions with very similar temperature dependence. Unfortunately, relatively few observations of the $\Delta n=1$ transitions of the beryllium-like ions are available, and detailed studies of the relative intensities of these lines have not been undertaken.

\section{(ii) Temperature}

Walker (1972) has reviewed earlier studies of temperature sensitive line ratios. Three types of ratios have been studied, the ratio of $2 s-3 p$ and $2 s-2 p$ lines in lithium-like ions, the ratio of $L \alpha$ and $L \beta$ in hydrogenic ions, and the ratio of the lithium-like satellite lines and helium-like line intensities.

Heroux et al. (1972) and Malinovsky and Heroux (1973) have carried out measurements of the line ratios for the lithium-like ions $\mathrm{O}$ VI, $\mathrm{Ne}$ VIII, and $\mathrm{Mg}$ XI. These authors find that the temperatures derived from the relative intensity of the $2 s-3 p$ and $2 s-2 p$ lines may be somewhat misleading if the lines are emitted over a broad temperature range.

Malinovsky and Heroux found that the observed $\mathrm{Ne}$ VIII and $\mathrm{Mg}$ xI line ratios are in good agreement with the theoretical ratios predicted using a coronal thermal model.

Rugge and Walker (1974) have analyzed the relative intensities of the $L \alpha$ and $L \beta$ lines of $\mathrm{O}$ VIII, $\mathrm{Mg}$ XII, and Si XIV, using a recent theoretical study of this line ratio carried out by Hutcheon and McWhirter (1973). Using a coronal temperature model Rugge and Walker were able to make a detailed comparison of the observed line ratios with the theory of Hutcheon and McWhirter. The predicted ratio of $\mathrm{L} \alpha$ to $\mathrm{L} \beta$ for $O$ VIII derived from the coronal model was $10.8 \pm 1.6$, while the observed ratio was found to be $10.5 \pm 0.5$. Good agreement was also found for the observed and predicted Mg XI and Si XIV ratios. However, Rugge and Walker found that the temperature predicted assuming an isothermal plasma differed from the calculated temperature of most efficient emission by $\sim 7 \times 10^{5} \mathrm{~K}$ for $\mathrm{O} \mathrm{VIII}$, by $1.6 \times 10^{6} \mathrm{~K}$ for $\mathrm{Mg} \mathrm{XI}$, and by $3.6 \times 10^{6} \mathrm{~K}$ for Si XIV. In each case, the 'predicted' temperatures were low.

These results suggest that the prediction of coronal temperatures from 'temperature sensitive' line ratios must be undertaken with considerable caution, unless there is other evidence that the observed lines were emitted from an isothermal structure. 


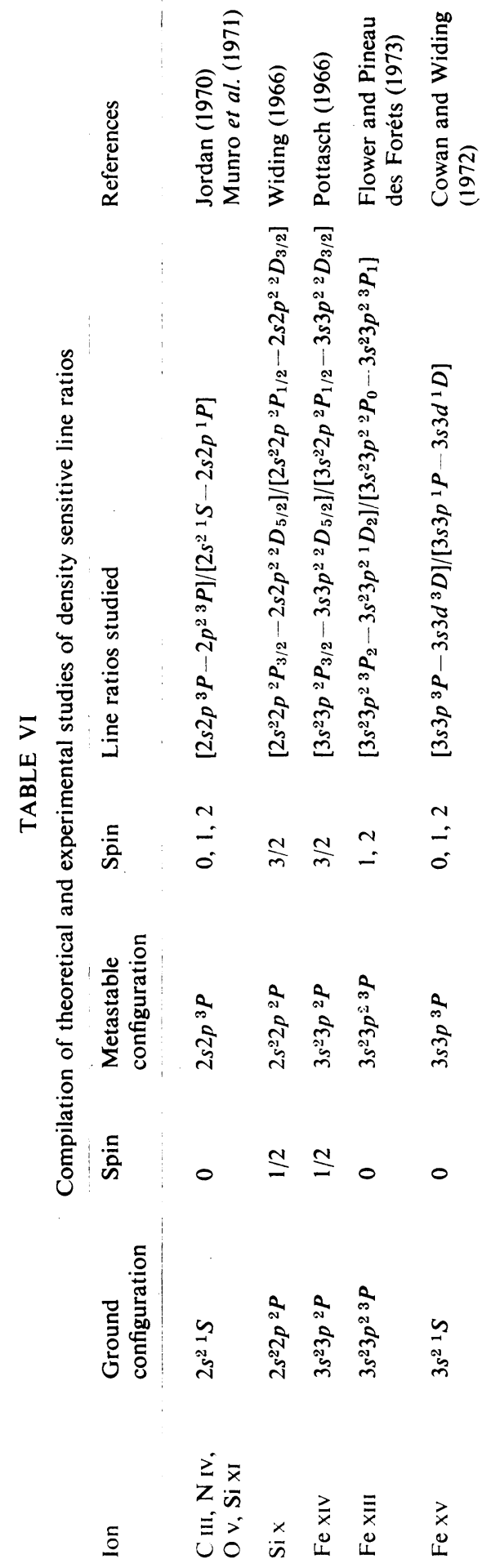


(iii) Non-Thermal Excitation

Two spectroscopic techniques have been suggested for the study of non-thermal excitation; the observation of non-autoionizing satellite lines in lithium-like ions, and the observation of K- $\alpha$ lines. Gabriel (1972) has discussed the observations of nonautoionizing satellite lines, and the calculation of the inner shell excitation rates which result in their production.

Phillips and Neupert (1973) have calculated the intensity expected for the $K-\alpha$ lines of sulfur, argon, calcium, and iron for thermal and non-thermal solar X-ray events. They have observed $\mathrm{K}-\alpha$ line emission from Fe XVII-XX during a large flare, and find that their theory predicts reasonable values of the emission measure.

\section{(c) CONSTRUCTION OF CORONAL MODELS}

Jefferies et al. (1972a) have developed a general method for the analysis of line emission from an optically thin gas, and applied this method to coronal forbidden line intensities (Jefferies et al., 1972b, c). Using the formalism developed by Jefferies et al., we may express the flux in a permitted coronal X-ray line as

$$
F_{Z z i j}=\varepsilon\left(\lambda_{i j}\right) a_{\mathrm{H}} A_{Z} \iint \mathrm{d} S N_{e} \iint \mathrm{d} n_{e} \mathrm{~d} T_{e} \mu\left(T_{e}, n_{e}\right) a_{z}\left(T_{e}\right) J\left(T_{e}\right) n_{e}
$$

We have separated the integrals over the area $S\left(x_{1}, x_{2}\right)$ which is unresolved by the telescope, and along the line of sight, $x_{3}$. Following Jefferies et al., we introduce the distribution function for the coronal plasma, $\mu\left[T_{e}\left(x_{v}\right), n_{e}\left(x_{v}\right)\right]$ which describes the density and temperature of the plasma, and replace the integral over $x_{3}$ by a double integral over this distribution function in a unit column. The quantity $\varepsilon\left(\lambda_{i g}\right)$ is the efficiency function of the spectroheliograph.

The function $\mu$ represents the fraction $(\mathrm{d} N)$ of the total number of electrons in a unit column $\left(N_{e}\right)$ which are in neighborhoods where temperature and density are given by $T_{e}$ and $n_{e}$.

$$
\mathrm{d} N\left(T_{e}, n_{e}, x_{1}, x_{2}\right)=N_{e} \mu\left(T_{e}, n_{e}, x_{1}, x_{2}\right) \mathrm{d} n_{e} \mathrm{~d} T_{e} .
$$

We may also introduce the partial distribution functions $\phi\left(T_{e}\right)$ and $\psi\left(T_{e}\right)$.

$$
\phi\left(T_{e}\right)=\int \mu\left(T_{e}, n_{e}\right) \mathrm{d} n_{e},
$$

and

$$
\psi\left(n_{e}\right)=\int \mu\left(T_{e}, n_{e}\right) \mathrm{d} T_{e}
$$

The function $J\left(T_{e}\right)$ [or $J\left(T_{e}, n_{e}\right)$ if the line in question is density dependent] contains the dependence of the excitation function on atomic parameters peculiar to the line being observed, and can be derived from Equations (5) and (12).

Equation (13) is an integral equation, with the kernel $A_{Z} a_{z}\left(T_{e}\right) J\left(T_{e}\right)$ which we can 
calculate, and the value $F$, which we can measure. In order to develop a set of techniques to determine $\mu\left(T_{e}, n_{e}\right)$, let us assume that there is a functional relationship between temperature and density, i.e.:

$$
n_{e}=n_{e}\left(T_{e}\right) .
$$

We may then write Equation (13) as

$$
F_{Z z i j}=\varepsilon\left(\lambda_{i j}\right) a_{\mathrm{H}} A_{Z} \iint \mathrm{d} S N_{e} \int \mathrm{d} T_{e} n_{e}\left(T_{e}\right) \phi\left(T_{e}\right) a_{z}\left(T_{e}\right) J\left(T_{e}\right) .
$$

The integral equation for $F_{Z z i j}$ defined by Equation (14) can, in principle, be solved to determine the temperature distribution of the emission measure; provided that observations including a sufficient number of lines with differing temperature dependence are available. The coronal models I discuss in Section III were derived by solving Equation (14), using various approximation procedures. The spatial integral must be extended over either individual active regions, or over the entire disk for the observations presently available, because of their low spatial resolution.

Pneuman (1972, 1973) and Kopp (1972) have pointed out the critical role that the magnetic field plays in the structure of coronal active regions, because of its predominant influence on the thermal conductivity. If observations of sufficient spatial (as well, of course, as spectral) resolution are available, we can introduce the dependence of $\phi$ (or of $\mu$ and $\psi$ ) on the magnetic field $B$ explicitly,

$$
F_{Z z i j}=\varepsilon\left(\lambda_{i j}\right) a_{\mathrm{H}} A_{Z} \int \mathrm{d} u \int \mathrm{d} s \int \mathrm{d} T_{e} n_{e}\left(T_{e}, B\right) \phi\left(T_{e}, B\right) a_{z}\left(T_{e}\right) J\left(T_{e}\right),
$$

where $s$ is a coordinate along the field line, and $u$ a coordinate transverse to the field lines. We may think of $u$ and $s$ as a set of solar B-L coordinates, much as those used to describe the terrestrial radiation belts. As Pneuman (1972) and Jordan (1975) point out, the coronal plasma will be nearly isothermal along a given field line, allowing simplifying assumptions to be made in the solution of Equation (15).

\section{Coronal Models}

Coronal models of the structure of active regions developed from X-ray observations have been limited because of the low spatial resolution of the spectroscopically resolved data presently available. Consequently, the models developed have emphasized the temperature structure and composition of the corona. The problem of the coronal energy balance has not been treated. A number of studies using EUV observations have been used to develop models of the energy balance of the transition region (Dupree, 1972; Munro and Withbroe, 1972; Withbroe and Gurman, 1973). Kopp (1972), Withbroe and Gurman (1973), and Gurman and Withbroe (1974) have summarized these results, and discussed the effect of magnetic fields on coronal structure. These authors find an increased 'coronal temperature' overlying active regions, and 
an increased conductive flux from the corona into the transition region. However, the lack of temperature discrimination for coronal temperatures above $\sim 2.5 \times 10^{6} \mathrm{~K}$ with EUV lines prevents these models from distinguishing between an isothermal corona, and a corona with thermal structures extending to higher temperatures beyond the most probable coronal temperature (i.e., the temperature at which the coronal emission measure is at a maximum). The analysis of the coronal X-ray spectra of active regions demonstrates that coronal material is present at considerably higher temperatures than the 'coronal active region temperature of $\sim 2.5-3 \times 10^{6} \mathrm{~K}$ ' which is derived from studies of the coronal EUV spectrum.

\section{(a) DEVELOPMENT OF CORONAL THERMAL MODELS}

Batstone et al. (1970) and Evans and Pounds (1968) developed the first thermal models, using coronal X-ray observations. They assumed that the emission function could be approximated by a step function, and found that coronal material as hot as $6-8 \times 10^{6} \mathrm{~K}$ must be present in order to explain their observations. Chambe (1971) developed a technique using an analytical expression for the emission measure function which has a maximum at a temperature $T_{0}$ (assumed to be near $2 \times 10^{6} \mathrm{~K}$ ) and falls off with temperature as $10^{-T / T_{1}}$. A study of the heating of the corona by Vil'koveskii (1972) shows that this is a reasonable thermal model based on the assumption that the mechanical heating is proportional to the square of the electron density. Vil'koveskii finds the parameters $T_{0}$ and $T_{1}$ to be $\sim 1.5 \times 10^{6}$ and $1 \times 10^{6} \mathrm{~K}$ for quiet regions, and $2.5 \times 10^{6}$ and $3 \times 10^{6} \mathrm{~K}$ for active regions. The model found by Chambe from observations requires $T_{0}=1.5 \times 10^{6} \mathrm{~K}$ and $T_{1}$ between 1.5 and $3.0 \times 10^{6} \mathrm{~K}$.

Walker (1972), Acton et al. (1972), Bonnelle et al. (1973), and Walker et al. (1974a, b) have developed models of the coronal emission measure, using the approach of Chambe. Acton et al. used the resonance lines of $\mathrm{O}$ VII and Ne IX, and were able to derive thermal models for a number of active regions, since their spectrometer was collimated to $1.7^{\prime}$ in one direction. They were able to map the emission of individual active regions by scanning the collimator across the solar disk in nearly orthogonal directions. The result of one of these scans is shown in Figure 6. The observations were fitted with an emission measure model $M(T)$ :

$$
M(T)=C \times 10^{-T / T_{1}} \mathrm{~cm}^{-3}\left(10^{6} \mathrm{~K}\right)^{-1} .
$$

The parameters found for each active region, and for the general corona are presented in Table VII. The total emission measure above $10^{6} \mathrm{~K}$, and the 'average' emission temperature of the $\mathrm{O}$ VII and $\mathrm{Ne}$ IX lines, are also given in Table VII.

Bonnelle et al. carried out a similar observation using the resonance lines of $\mathbf{M g ~ X I}$ and $\mathrm{Mg}$ XII, and satellite lines of $\mathrm{Mg} \mathrm{x}$. These authors developed a thermal model with $C=5 \times 10^{48} \mathrm{~cm}^{-3}\left(10^{6} \mathrm{~K}\right)^{-1}$, and $T_{1}=2.6 \times 10^{6} \mathrm{~K}$.

Walker (1972) and Walker et al. (1974a, b) have developed models using the resonance lines of O viI, O viII, Ne Ix, Ne x, Mg XI, Mg XII, Al XII, Al XIII, Si XIII, and Si XIV. They find an excellent fit to the model proposed by Chambe and Vil'koveskii, with $T_{0}=2.5 \times 10^{6} \mathrm{~K}$ and $T_{1}=3.5-4.0 \times 10^{6} \mathrm{~K}$. The models developed by Walker 


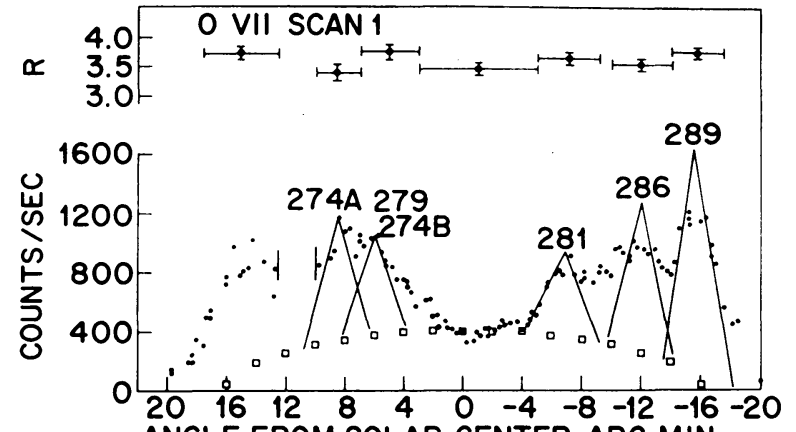

(a) ANGLE FROM SOLAR CENTER ARC MIN
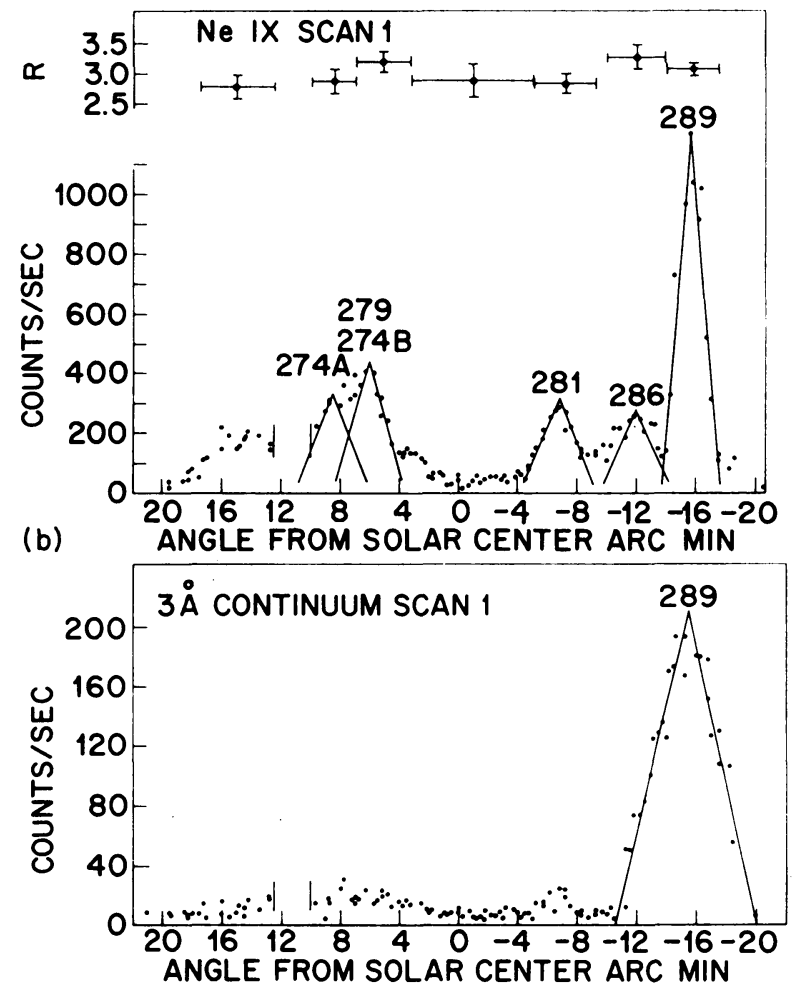

(c)

Fig. 6. Variation of the coronal X-ray intensity across the solar disk observed by Acton et al. (1972) with a one dimensional collimator. A second scan approximately orthogonal to the scan shown here allowed a map of the coronal structure to be constructed, and the models given in Table VII.

et al. are shown in Figures 7 and 8 . The small values of the parameter $\chi^{2}$ demonstrate the significance of the fit. From Figure 7, we see that a model with a temperature cutoff will fit the observations since the hottest line included in the model is $\mathrm{Mg}$ XI. In Figure 8 , we note that the model must include material in excess of $8 \times 10^{6} \mathrm{~K}$ in order to fit the Si XIV observations. 
The model of an isothermal corona developed from the analysis of EUV observations must be modified for active regions. For coronal holes, and for the quiet corona, where magnetically open geometries introduce the solar wind as an energy loss term (Pneuman, 1973), the coronal structure can be reasonably fit by an isothermal model,

TABLE VII

Non-isothermal (exponential) model

\begin{tabular}{llcccc}
\hline Plage region & $\begin{array}{l}T_{1} \\
\left(10^{6} \mathrm{~K}\right)\end{array}$ & $\mathrm{C}^{\mathrm{a}}$ & $\begin{array}{l}\mathrm{EM}_{\mathrm{tot}}{ }^{\mathrm{b}} \\
\left(10^{47} \mathrm{~cm}^{-3}\right)\end{array}$ & $\begin{array}{l}\left\langle\boldsymbol{T}_{\mathrm{O}_{\mathrm{NII}}}\right\rangle \\
\left(10^{6} \mathrm{~K}\right)\end{array}$ & $\begin{array}{l}\left\langle\boldsymbol{T}_{\mathrm{Ne}_{\mathrm{IX}} \mathrm{X}}\right\rangle \\
\left(10^{6} \mathrm{~K}\right)\end{array}$ \\
289 & & & & & \\
$279,274 \mathrm{~B}$ & 3.15 & 3 & 23 & 1.95 & 3.10 \\
281 & 1.80 & 6 & 13 & 1.80 & 2.70 \\
$274 \mathrm{~A}$ & 1.50 & 8 & 12 & 1.70 & 2.55 \\
286 & 1.25 & 18 & 16 & 1.65 & 2.40 \\
265,266 & 1.15 & 24 & 16 & 1.60 & 2.30 \\
Gen. corona $^{\mathrm{c}}$ & 0.95 & 42 & 15 & 1.50 & 2.20 \\
& 0.60 & 170 & 170 & 1.25 & 1.70
\end{tabular}

a Units: $10^{48} \mathrm{~cm}^{-3}\left(10^{6} \mathrm{~K}\right)^{-1}$.

b) Emission measure above $10^{6} \mathrm{~K}$.

" All values refer to coronal hemisphere facing the Earth.

Taken from Acton et al. (1972).

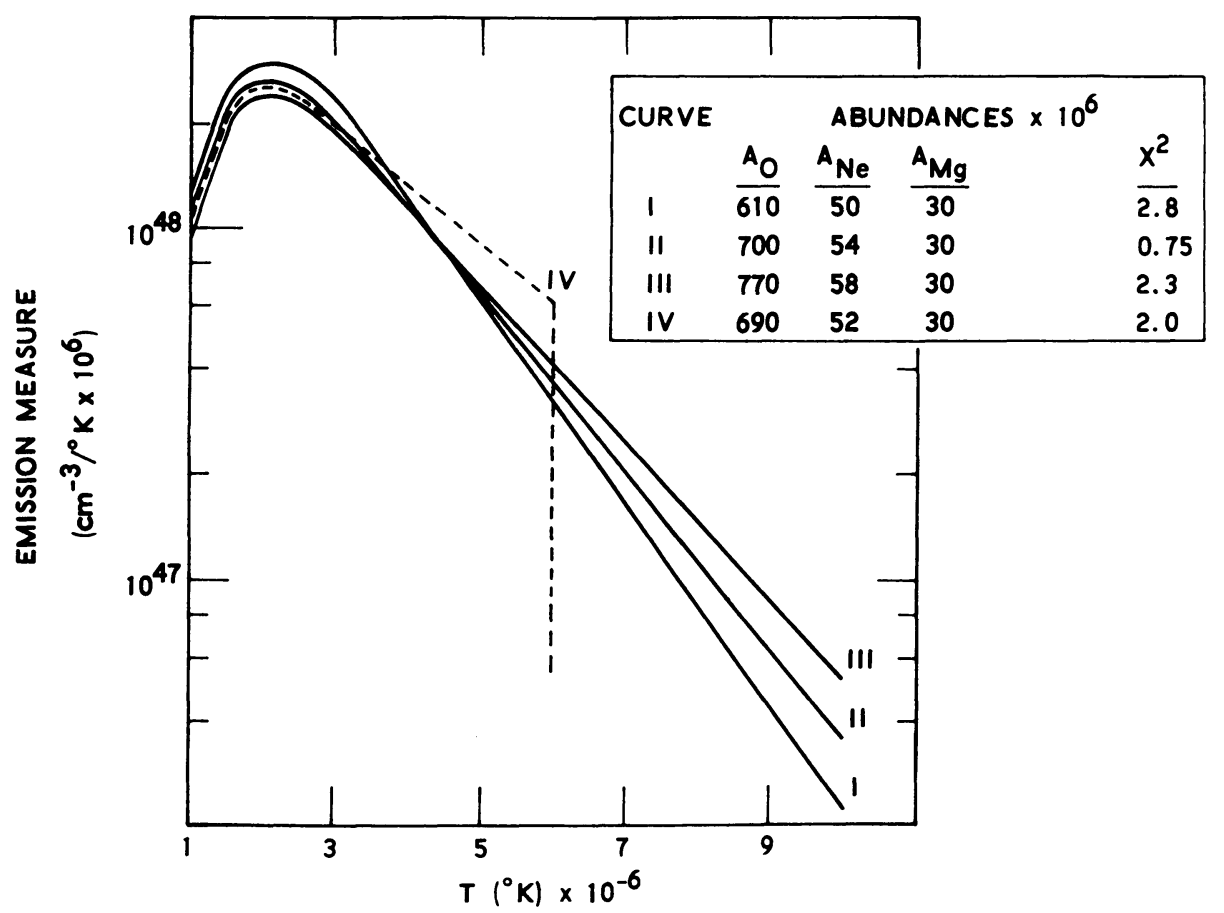

Fig. 7. Coronal Model constructed from the resonance lines of $\mathrm{O}$ VII, $\mathrm{O}$ VIII, $\mathrm{Ne} I x, \mathrm{Ne} X$, and $\mathrm{Mg} \mathrm{XI}$. Note that a model with no material above $6 \times 10^{6} \mathrm{~K}$ also fits the observations. A model cutoff above $5.0 \times 10^{6} \mathrm{~K}$ does not, however, fit the observations. 


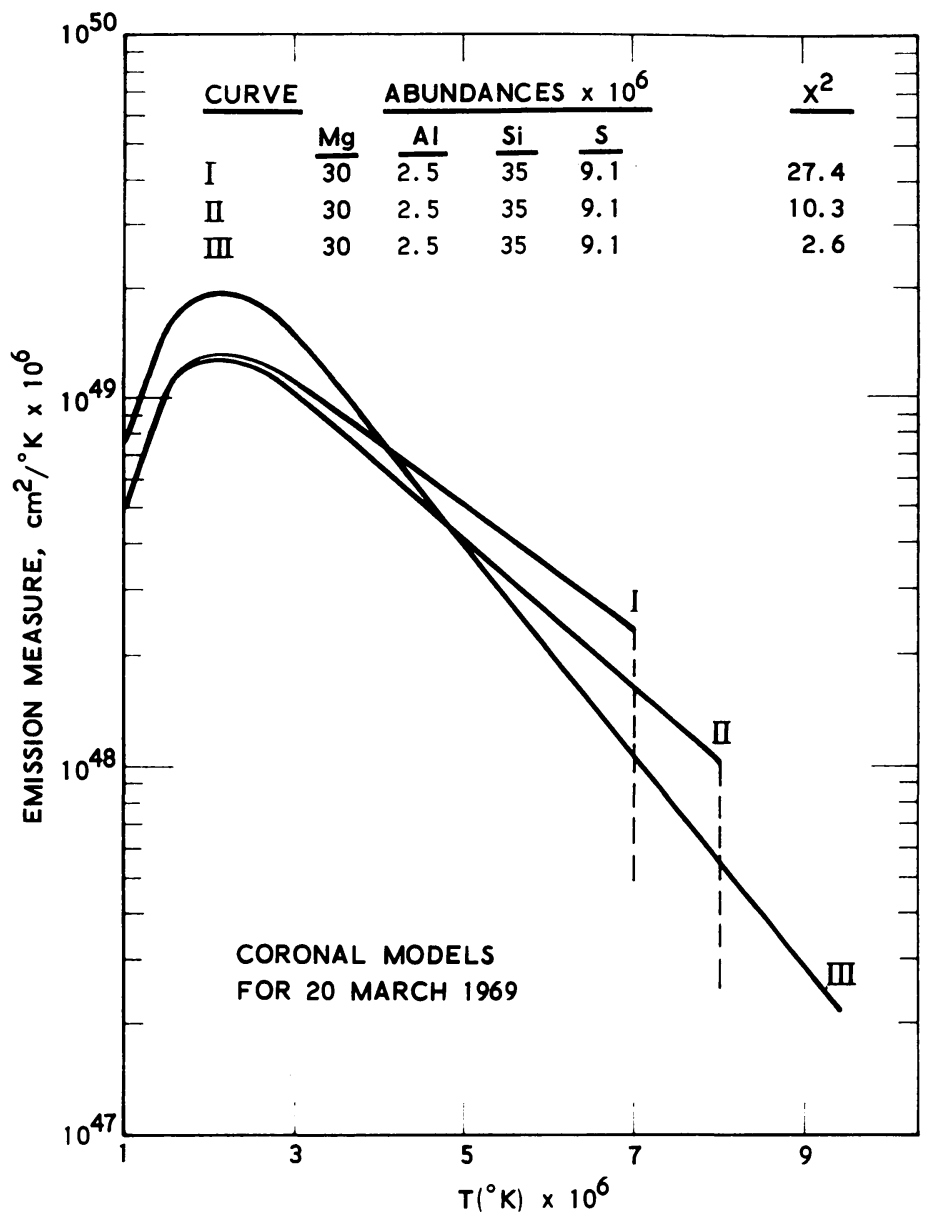

Fig. 8. Coronal Model derived from the resonance lines of $\mathrm{Mg}$ IX, $\mathrm{Mg} \mathrm{X}, \mathrm{Al}$ XII, Al XIII, Si XIII, $\mathrm{Si}$ xIv, $\mathrm{S} \times \mathrm{x}$. Note that material at temperatures above $8 \times 10^{6} \mathrm{~K}$ must be included in the model in order to fit the hot Si XIV and $\mathrm{S} x \mathrm{~V}$ lines.

with $T \sim 1.0 \times 10^{6} \mathrm{~K}$ (Munro and Withbroe, 1972) for coronal holes and $\sim 1.5 \times 10^{6} \mathrm{~K}$ for the quiet corona (Withbroe and Gurman, 1973). Acton et al. find that the general coronal emission measure does indeed fall off very rapidly above the temperature of maximum emission measure $\left(T_{0} \sim 1.5 \times 10^{6} \mathrm{~K}\right)$ with $T_{1} \sim 0.3-1.0 \times 10^{6} \mathrm{~K}$. However, for active regions, the emission measure falls off much more slowly, with $T_{1} \sim 1.5-4.0 \times 10^{6} \mathrm{~K}$, and models with an isothermal corona, even with temperatures as high as $2.5-3.5 \times 10^{6} \mathrm{~K}$ (Withbroe and Gurman, 1973; Noyes et al., 1970), cannot account for the X-ray observations.

The high temperatures required to account for the coronal X-ray flux may be an indication of a heating source in addition to the dissipation of mechanical waves, the source generally assumed to be responsible for coronal heating (Kopp and Kupers, 1968). Tucker (1973), Sturrock (1972b), and Syrovatskii and Shmeleva (1972) have 
suggested that the dissipation of magnetic energy by ohmic heating in current sheets may contribute to the thermal balance of active regions. Highly spatially, as well as spectrally resolved observations will be required to demonstrate that magnetic heating is taking place in the corona, if it is, indeed, an important component in the thermal energy balance.

(b) CORONAL ABUNDANCES

Walker et al. (1974a, b, c) have derived coronal abundances from the models given in Figures 7 and 8 . The abundances derived by Walker et al. are compared with the coronal abundances derived with transition region observations by Withbroe (1971a), Dupree (1972), and Malinovsky and Heroux (1972), and with photospheric abundances compiled by Withbroe (1971a) and Cameron (1974) in Table VIII. In general, the agreement is good; however, there is a significant difference for the neon abundance. Cameron's neon abundance is obtained from cosmic ray observations. Since the neon lines used by Walker et al. (1974b) in their analysis were quite strong, and were excited near the mid-range of temperatures in their model I believe their neon results to be quite reliable, and the difference may, therefore, be significant. In the case of the sulfur and argon abundances, the lines analyzed were near the high temperature end of their model, and are thought to be somewhat less reliable than the other abundances. Consequently, we do not regard the difference between the coronal and the photospheric computations to be significant for these elements. The oxygen abundance found by Withbroe and by Malinovsky and Heroux for the transition region is low, however, Dupree's results appear to support a higher oxygen abundance.

TABLE VIII

Comparison of relative abundances Abundance $\times\left(10^{6}\right)$

\begin{tabular}{|c|c|c|c|c|c|c|}
\hline \multirow[t]{2}{*}{ Element } & \multirow{2}{*}{$\frac{\text { Active region }}{\text { Walker } \text { et al. }}$} & \multicolumn{3}{|c|}{ Transition region } & \multirow{2}{*}{$\begin{array}{l}\text { Photospheric } \\
\text { Withbroe }\end{array}$} & \multirow{2}{*}{$\begin{array}{l}\text { Solar system } \\
\text { Cameron }\end{array}$} \\
\hline & & Withbroe & Dupree & $\begin{array}{l}\text { Malinovsky } \\
\text { and Heroux }\end{array}$ & & \\
\hline C & - & 420 & 350 & - & 350 & 370 \\
\hline $\mathbf{N}$ & 90 & 89 & 150 & - & 115 & 117 \\
\hline $\mathrm{O}$ & 700 & 450 & 595 & 350 & 676 & 676 \\
\hline $\mathrm{Ne}$ & 54 & 28 & 27 & 88 & - & 108 \\
\hline $\mathrm{Na}$ & 1.7 & 2.3 & 1.9 & - & 1.7 & 1.9 \\
\hline $\mathbf{M g}$ & 30 & 35 & 30 & 50 & 35 & 33 \\
\hline $\mathrm{Al}$ & 2.5 & 2.3 & 3.5 & - & 2.5 & 2.7 \\
\hline $\mathrm{Si}$ & $35^{\mathrm{a}}$ & $35^{\mathrm{a}}$ & $35^{\mathrm{a}}$ & $35^{\mathrm{a}}$ & 35 & 31.6 \\
\hline $\mathbf{S}$ & 9 & 11 & 20 & 5 & 16 & 16 \\
\hline $\mathrm{Ar}$ & 6 & 4.5 & - & - & - & 3.7 \\
\hline $\mathrm{Fe}$ & 26 & 35 & 20 & 20 & $25^{b}$ & 26 \\
\hline
\end{tabular}

a The coronal abundance values have been normalized relative to the silicon abundance, which was assumed to be $35 \times 10^{-6}$.

b In a recent analysis, Smith and Whaling (1973) find a value of $25 \times 10^{-6}$ for the iron abundance, in agreement with the earlier result of Withbroe. 
In conclusion, it would appear that the results of Table VIII support a uniform abundance structure for the solar atmosphere.

\section{(c) CORONAL DENSITY STRUCTURE}

The X-ray analyses of coronal density structure have, so far, resulted only in upper limits. The analyses of density sensitive EUV lines by Munro et al. (1971), Jordan (1971), Widing (1966), and others (see Table VI) have resulted in density models which predict densities of $\sim 5 \times 10^{8} \mathrm{~cm}^{-3}$ in the quiet corona, and densities as high as $2.5 \times 10^{9} \mathrm{~cm}^{-3}$ in active regions. These models are consistent with the X-ray upper limits, and with other observations. Withbroe (1971b) has shown that the intensity of the $\mathrm{Mg} \mathrm{X}$ resonance line is a good indicator of coronal density, once properly calibrated. Withbroe et al. (1971) and Withbroe and Gurman (1973) have developed coronal density maps using this technique, and Gurman et al. (1974) have discussed the correlation of coronal density and photospheric magnetic intensity using this technique.

\section{(d) THE STRUCTURE OF CORONAL ACTIVE REGIONS}

The poor spatial resolution of the presently available spectroscopically resolved coronal X-ray observations makes it difficult to study the physical structure of active regions. Parkinson (1973a, b) has used two types of observations, high spectral resolu-

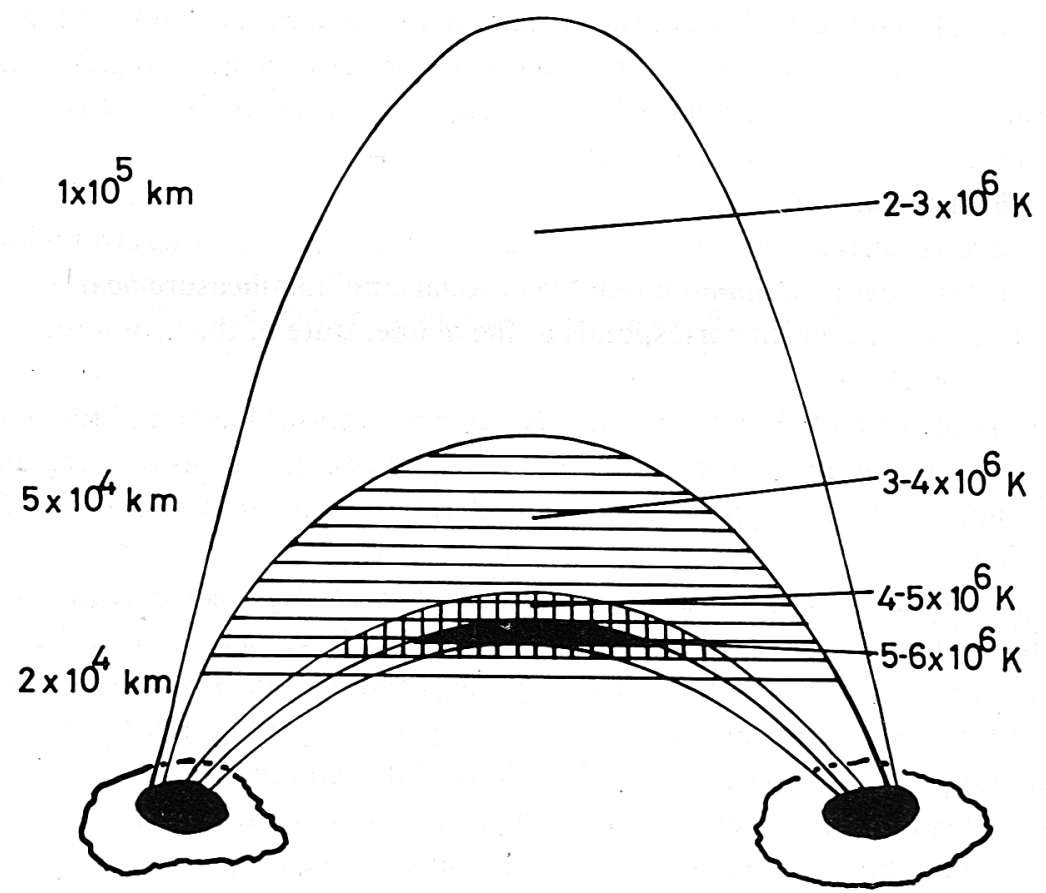

Fig. 9. Model of a coronal active region derived by Parkinson (1973a, b) from OSO 5 spectroheliograms and rocket spectra of active regions. 
tion studies with low spatial resolution, and moderate spectral resolution studies of coronal structures from OSO 5 with 1'-2' resolution (Parkinson and Pounds, 1971) to construct the schematic model shown in Figure 9. This model resembles the X-ray loop structures observed on Skylab (Vaiana, 1974; Underwood, 1974) with high resolution X-ray telescopes.

Pneuman $(1972,1973)$ has analyzed the structure of coronal active regions in open and closed magnetic structure, and points out that coronal material should be isothermal along magnetic field lines, because of the large thermal conductivity along field lines. This suggests that we may think of an active region as a collection of isothermal flux tubes, with the tubes at lower altitude being higher in temperature. As the flux tube approaches the photosphere, the isothermal approximation breaks down, and the transition region, as described by Kopp (1972) occurs. The temperature structure along each flux tube would indeed have an 'isothermal' corona, coupled to the transition region, however, taken together, the flux tubes result in a thermal structure for the active region which is described by Figures 7 and 8 . The study of this structure may require very high $\left(<10^{\prime \prime}\right)$ spatial resolution. Jordan (1975) has discussed the analytical and observational evidence for the 'multiple flux tube' model of coronal arch (or loop) structures elsewhere in these proceedings.

\section{Conclusions}

We have found that the analysis of the coronal X-ray spectrum has resulted in coronal thermal models which show a nearly isothermal corona over quiet regions, in agreement with analyses of the coronal EUV spectrum. However, analyses of active region spectra (or of whole disk spectra which are dominated by active regions) result in models which contain material as hot as $8-10 \times 10^{6} \mathrm{~K}$, in contradiction to the "isothermal' corona derived from the analysis of the EUV spectra of active regions. The $X$-ray results do predict a maximum in the coronal emission measure near $\sim 2-3 \times 10^{6}$ $\mathrm{K}$, for active regions, which corresponds to the temperature of the 'isothermal' corona found by the EUV analyses.

The study of coronal abundances with X-ray observations has found relative abundances which are in good agreement with photospheric and transition region abundances, lending further support to the view that the solar atmosphere is uniform in composition.

The study of the structure and energy balance of active regions will require observations with better spatial resolution than those which have been analyzed up to this time. The S-056 and S-054 X-ray telescope experiments on Skylab (Vaiana et al., 1973; Underwood, 1974), and the OSO-7 X-ray spectroheliograph (Neupert et al., 1974) should provide much of the observational data required for these analyses. All three instruments make use of filters to provide wavelength (and consequently temperature) discrimination, so that accurate calculations of the shape of the coronal spectrum will be required in order to carry out the type of analysis of coronal structure outlined in Section II (c). 


\section{References}

Acton, L. W., Catura, R. C., Meyerott, A. J., Wolfson, C. J., and Culhane, J. L.: 1972, Solar Phys. 26, 183.

Batstone, R. M., Evans, K., Parkinson, J. H., and Pounds, K. A.: 1970, Solar Phys. $13,389$.

Bely, O.: 1966a, Ann. Astrophys. 29, 683.

Bely, O.: 1966b, Proc. Phys. Soc. 88, 587.

Bely, O. and Bely, F.: 1967, Solar Phys. 2, 285.

Bely, O. and Petrini, D.: 1970, Astron. Astrophys. 6, 318.

Bonnell, C., Senemand, C., Senemand, G., Chambre, G., Guionnet, M., Heroux, J. C., and Michaud, R.: 1973, Solar Phys. 29, 341.

Burgess, A., Hummer, D. G., and Tully, J. A.: 1970, Phil. Trans. Roy. Soc. London A266, 225.

Burke, P. G., Hubbert, A., and Robb, W. D.: 1972, J. Phys. B5, 37.

Burke, P. G., Tait, J. H., and Lewis, B. A.: 1966, Proc. Phys. Soc. 87, 209.

Cameron, A. G. W.: 1974, Space Sci. Rev. 15, 121.

Chambe, G.: 1971, Astron. Astrophys. 12, 210.

Cowan, R. D. and Widing, K. G.: 1972, Astrophys. J. 180, 285.

Culhane, J. L.: 1969, Monthly Notices Roy. Astron. Soc. 144, 375.

Culhane, J. L. and Acton, L. W.: 1974, Ann. Rev. Astron. Astrophys. 12, 359, Ann. Rev. Inc. Palo Alto.

Doschek, G. A.: 1972, Space Sci. Rev. 13, 765.

Dupree, A. K.: 1972, Astrophys. J. 178, 527.

Evans, K. and Pounds, K. A.: 1968, Astrophys. J. 152, 313.

Flower, D. R.: 1971, J. Phys. B4, 697.

Flower, D. R. and Launay, J. M.: 1972, J. Phys. B5, L207.

Flower, D. R. and Pineau des Forets, G.: 1973, Astron. Astrophys. 24, 181.

Gabriel, A. H.: 1972, Monthly Notices Roy. Astron. Soc. 160, 99.

Gabriel, A. H. and Jordan, Carole: 1972, in E. W. McDaniel and M. R. C. McDowell (eds.), Case Studies in Atomic Collision Physics, Vol. II, North-Holland Publ. Co., Amsterdam, p. 211.

Gabriel, A. H. and Jordan, Carole: 1973, Astrophys. J. 186, 327.

Gabriel, A. H. and Paget, T. M.: 1972, J. Phys. B5, 673.

Garstang, R. H.: 1969, Publ. Astron. Soc. Pacific 81, 488.

Grineva, Y. I., Karev, V. I., Korneev, V. V., Krutov, V. V., Mandelstam, S. L., Varnshtein, L. A., Vaisiljev, B. N., and Zitnik, I. A.: 1973, Solar Phys. 29, 441.

Gurman, J. B., Withbroe, G. L., and Harvey, J. W.: 1974, Solar Phys. 34, 105.

Heroux, L. and Cohen, M.: 1971, Phil. Trans. Roy. Soc. London A270, 99.

Heroux, L., Cohen, M., and Malinovsky, Monique: 1972, Solar Phys. 23, 369.

Hutcheon, R. J. and McWhirter, R. W. P. : 1973, J. Phys. B6, 2268.

Jefferies, J. T., Orrall, G. Q., and Zirker, J. B.: 1972a, Solar Phys. 22, 307.

Jefferies, J. T., Orrall, G. Q., and Zirker, J. B.: 1972b, Solar Phys. 22, 317.

Jefferies, J. T., Orrall, G. Q., and Zirker, J. B.: 1972c, Solar Phys. 22, 327.

Johnson, W. D. and Kunze, H. J.: 1971, Phys. Rev. A4, 962.

Jordan, Carole: 1966, Monthly Notices Roy. Astron. Soc. 132, 463.

Jordan, Carole: 1969, Monthly Notices Roy. Astron. Soc. 142, 499.

Jordan, Carole: 1970, Monthly Notices Roy. Astron. Soc. 149, 1.

Jordan, Carole: 1971, in C. de Jager (ed.), Highlights in Astronomy, D. Reidel Publ. Co., Dordrecht, Holland, p. 519

Jordan, Carole: 1975, This volume, p. 109.

Kopp, R. A.: 1972, Solar Phys. 27, 373.

Kopp, R. A. and Kupers, M.: 1968, Solar Phys. 4, 212.

Landini, M. and Monsignori Fossi, B. C.: 1970, Astron. Astrophys. 6, 468.

Landini, M. and Monsignori Fossi, B. C. : 1972, Astron. Astrophys. Suppl. 7, 291.

Loulergue, M.: 1971, Astron. Astrophys. 15, 126.

Loulergue, M.: 1973, Private communication.

Loulergue, M. and Nussbaumer, H.: 1973, Astron. Astrophys. 24, 312.

Malinovsky, Monique and Heroux, L.: 1973, Astrophys. J. 181, 1009. 
Mewe, R.: 1972, Solar Phys. 22, 459.

Munro, R. H., Dupree, A. K., and Withbroe, G. L.: 1971, Sol. Phys. 19, 347.

Neupert, W. M., Thomas, R. J., and Chapman, R. D. : 1974, Solar Phys. 34, 349.

Noyes, R.: 1971, Ann. Rev. Astron. Astrophys. 2, 209.

Noyes, R., Withbroe, G. L., and Kirschner, R. P.: 1970, Solar Phys. 11, 388.

Parkinson, J. H.: 1973a, Solar Phys 28, 137.

Parkinson, J. H.: 1973b, Solar Phys. 28, 489.

Parkinson, J. H.: 1975, This volume, p. 45.

Parkinson, J. H. and Pounds, K. A.: 1971, Solar Phys. 17, 146.

Phillips, K. J. H. and Neupert, W. M.: 1973, Solar Phys. 32, 209.

Pneuman, G. W.: 1972, Astrophys. J. 177, 793.

Pneuman, G. W.: 1973, Solar Phys. 28, 247.

Pottasch, S. R.: 1964, Space Sci. Rev. 3, 816.

Pottasch, S. R.: 1966, Bull. Astron. Inst. Neth. 18, 237.

Pottasch, S. R.: 1967, Bull. Astron. Inst. Neth. 19, 113.

Rugge, H. R. and Walker, A. B. C.: 1970, Solar Phys. 15, 372.

Rugge, H. R. and Walker, A. B. C.: 1971, Solar Phys. 18, 244.

Rugge, H. R. and Walker, A. B. C.: 1974, Astron. Astrophys. 33, 367.

Sampson, D. H. and Parks, A. D.: 1974, Astrophys. J. Suppl. Series 28, 323.

Shore, B.: 1969, Astrophys. J. 158, 1205.

Smith, P. L. and Whaling, W.: 1973, Astrophys. J. 183, 313.

Sturrock, P. A.: 1972a, Solar Phys. 23, 438.

Sturrock, P. A.: 1972b, Progress Astron. Aeronaut. 30, 172.

Syrovatskii, S. I. and Shmeleva, O. P.: 1972, Soviet Astron-A.J. 16, 273.

Tondello, G. and McWhirter, R. W. P.: 1971, J. Phys. B4, 715.

Tully, J. A.: 1974, J. Phys. B7, 386.

Tucker, W. H.: 1973, Astrophys. J. 186, 285.

Tucker, W. H. and Koren, M.: 1971, Astrophys. J. 168, 283.

Underwood, J. H.: 1974, Paper presented at the Joint IAU-COSPAR Symp. 68 on 'Solar Gamma, Xand EUV Radiation', Buenos Aires, Argentina, 11-14 June, 1974 (unpublished).

Vaiana, G.: 1974, Paper presented at the Joint IAU-COSPAR Symp. 68 on 'Solar Gamma, X- and EUV Radiation', Buenos Aires, Argentina, 11-14 June, 1974 (unpublished).

Vaiana, G. S., Davis, J. M., Giacconi, R., Kreger, A. S., Silk, J. K., Timothy, A. F., and Zombeck, M.: 1973, Astrophys. J. Letters 185, 247.

Van Regemorter, H.: 1962, Astrophys. J. 136, 906.

Vil'koviskii, E. Ya.: 1972, Astron Zh. 49, 1125 (English translation Soviet Astron. - A.J. 16, 918.)

Walker, A. B. C.: 1972, Space Sci. Rev. 13, 672.

Walker, A. B. C. and Rugge, H. R.: 1971, Astrophys. J. 164, 181.

Walker, A. B. C., Rugge, H. R., and Weiss, K.: 1974a, Astrophys. J. 188, 423.

Walker, A. B. C., Rugge, H. R., and Weiss, K.: 1974b, Astrophys. J. 192, 169.

Walker, A. B. C., Rugge, H. R., and Weiss, K. : 1974c, Astrophys. J. 194, 471.

Walker, D. W.: 1974, J. Phys. B7, 97.

Widing, K. G.: 1966, Astrophys. J. 145, 380.

Withbroe, G. L.: 1971a, in K. B. Gebbie (ed.), The Menzel Symposium on 'Solar Physics, Atomic Spectra, and Gaseous Nebulae', NBS Special Publication 353, 127.

Withbroe, G. L.: 1971b, Solar Phys. 18, 458.

Withbroe, G. L., Dupree, A. K., Goldberg, L., Huber, C. E., Noyes, R. W., Parkinson, W. H., and Reeves, E. M.: 1971, Solar Phys. 21, 272.

Withbroe, G. L. and Gurman, J. B.: 1973, Astrophys. J. 183, 279. 